\title{
Balanced data assimilation for highly oscillatory mechanical systems
}

\author{
Gottfried Hastermann* \\ Maria Reinhardt ${ }^{\dagger}$ \\ Rupert Klein ${ }^{\ddagger}$ \\ Sebastian Reich ${ }^{\S}$
}

April 16, 2021

\begin{abstract}
Data assimilation algorithms are used to estimate the states of a dynamical system using partial and noisy observations. The ensemble Kalman filter has become a popular data assimilation scheme due to its simplicity and robustness for a wide range of application areas. Nevertheless, this filter also has limitations due to its inherent assumptions of Gaussianity and linearity, which can manifest themselves in the form of dynamically inconsistent state estimates. This issue is investigated here for balanced, slowly evolving solutions to highly oscillatory Hamiltonian systems which are prototypical for applications in numerical weather prediction. It is demonstrated that the standard ensemble Kalman filter can lead to state estimates that do not satisfy the pertinent balance relations and ultimately lead to filter divergence. Two remedies are proposed, one in terms of blended asymptotically consistent time-stepping schemes, and one in terms of minimization-based post-processing methods. The effects of these modifications to the standard ensemble Kalman filter are discussed and demonstrated numerically for balanced motions of two prototypical Hamiltonian reference systems.
\end{abstract}

Keywords. Data assimilation, ensemble Kalman filter, balanced dynamics, highly oscillatory systems, Hamiltonian dynamics, geophysics

AMS (MOS) subject classifications. 65C05, 62M20, 93E11, 62F15, 86A22

\footnotetext{
*Freie Universität Berlin, Institut für Mathematik, Arnimallee 6, D-14195 Berlin, Germany

${ }^{\dagger}$ Universität Potsdam, Institut für Mathematik, Karl-Liebknecht-Str. 24/25, D-14476 Potsdam, Germany

${ }^{\ddagger}$ Freie Universität Berlin, Institut für Mathematik, Arnimallee 6, D-14195 Berlin, Germany

$\S$ Universität Potsdam, Institut für Mathematik, Karl-Liebknecht-Str. 24/25, D-14476 Potsdam, Germany
} 
Balanced data assimilation

\section{Introduction}

A problem dating back as far as the advent of numerical weather prediction is the incorporation of physical observations into a dynamical model with more than one time scale. The famous first forecast of L. F. Richardson [40] failed due to the choice of an unbalanced initial condition gained from observations. In essence the observational data did not satisfy certain discrete energy balances and this triggered artificial oscillations in the pressure, ultimately leading to erroneous results. In the context of data assimilation, several solutions to the related problem of finding balanced initial data were proposed over the last decades. Lynch [41] suggested to apply a digital filter after every assimilation step to eliminate spurious fast oscillations, and this technique was adopted in the weather prediction context with some success. Strategies that incorporate the observational data in the model evolution in a gradual and smooth way instead of using all the information about the observation at one single point in time have been suggested for example in [7] and [6]. Kepert [31] proposed a method to overcome the issue of artificial balances triggered by localized Bayesian data assimilation. Here "localization" refers to approaches designed to avoid spurious long-range correlations by allowing the model state in a given grid point of a flow simulation to be influenced only by data found within a given maximum distance from it. Kepert suggested to localize the data filter in the streamfunction and velocity potential fields rather than in the velocity or momentum variables. With a similar goal, Gottwald [23] incorporated additional climatological information in the assimilation process in the sense of variance-limited Kalman filters, 24], so as to drive the model's level of imbalance towards its climatological mean.

In the context of variational data assimilation for slow-fast Hamiltonian systems the issue was addressed, e.g., by Cotter [16]. Variational methods, in contrast to the "filtering techniques" referred to in the last paragraph, aim to optimize the match between simulation and observation over an entire time window in the past, i.e., not only just at the time when the observations arrive. Cotter's approach differs from the ones cited above in that he explicitly uses an analytical fast-slow transformation of variables which he assumes can be derived from the structure of the system's Hamiltonian and which defines its relevant slow manifold. In this situation, he defines artifical Hamiltonian dynamics that drives a system state from arbitrary states towards close-by states on the slow manifold relatively quickly, but still on the slow time scale. He then suggests to use this artificial dynamics to constrain a variational data assimilation method (4DVAR) so as to produce a balanced state as the initial condition for the next forecast at the end of the data assimilation time window.

Here we propose two alternative approaches to addressing the balancing problem for data assimilation based on filtering techniques. The first approach relies on the ensemble-based Bayesian sequential data assimilation paradigm and is designed as a post-processing step in the filtering procedure that penalizes imbalances and is structurally 
similar to the 3DVAR method, [29]. Two alternatives for the computational implementation of this post-processing step are briefly discussed, comprising a Gauss-Newton minimization and a pseudo-time evolution.

The second approach proposed in this paper is, in contrast, incorporated in the forward simulation and relies on the ability of our asymptotically consistent numerical method to seamlessly represent balanced and unbalanced dynamics. In some aspects, this approach is similar to that of Cotter [16] in that we use analytical knowledge regarding the full oscillatory and a nearby reduced slow dynamics. Yet, our ansatz is not tied closely to Hamiltonian structure, and our balancing strategy is a direct part of the forward simulation rather than being incorporated in the data assimilation procedures. The key idea, first formulated in [3], is to first apply some known filtering technique for data assimilation that is not specifically designed to maintain physical balances, but to start the subsequent forward simulation by several time steps with a "blended model" that interpolates in a judicious way between the reduced slow and the full oscillatory dynamics. The discretization of the interpolating model family is dissipative with respect to the fast modes, while properly advancing the balanced modes. As a consequence, when the full dynamics becomes active after the blending time window, fast oscillations have been removed and nearly balanced conditions prevail throughout the remaining majority of the forward simulation time steps.

The rest of this section introduces the class of highly-oscillatory nonlinear finite dimensional test problems utilized in this paper, and discusses the failure of a Bayesian data assimilation procedure which has motivated our work. Section 2 describes the two balanced data assimilation approaches proposed in this paper. Section 3 compares the performance of several data assimilation techniques for the oscillatory test problem. Section 4 provides a summary and an outlook to future work.

\subsection{Model problem}

With atmospheric models in mind as a motivation, we propose numerical techniques that allow the user to follow the slow evolution of a system with multiple time scales starting from balanced initial data. In doing so, we restrict to the finite-dimensional setting in this paper, in line with Lorenz' seminal investigations of oscillatory systems and predictability in [37, 38] and, more specifically, with studies into the existence and properties of slow manifolds for multiple time scale Hamiltonian systems in [8, 39, 11].

In particular, we discuss sequential data assimilation for highly-oscillatory systems with Hamiltonian energy functional

$$
H^{\varepsilon}(q, p)=\frac{1}{2} p^{\mathrm{T}} p+\frac{1}{2 \varepsilon^{2}} g(q)^{\mathrm{T}} K g(q)+V(q)
$$

with momenta and coordinates $p, q \in \mathbb{R}^{N}$. Here $V: \mathbb{R}^{N} \rightarrow \mathbb{R}$ is a potential energy, $g: \mathbb{R}^{N} \rightarrow \mathbb{R}^{L}, L \leq N$ gives rise to rapid oscillations with a diagonal matrix of force constants $K=\operatorname{diag}\left(k_{1}, \ldots, k_{L}\right), k_{i}>0$, and $\varepsilon$ is a stiffness 
parameter satisfying $0<\varepsilon \ll 1$. The associated Hamiltonian equations of motion are then given by

$$
\begin{aligned}
& \dot{q}=p \\
& \dot{p}=-\varepsilon^{-2} G(q)^{\mathrm{T}} K g(q)-\nabla V(q),
\end{aligned}
$$

where $G(q):=D g(q) \in \mathbb{R}^{L \times N}$ denotes the Jacobian matrix of $g$ at $q$. These equations pose challenges in their numerical treatment as well as for sequential data assimilation techniques in the limit $\varepsilon \rightarrow 0$. We observe that solutions of (2) preserve the Hamiltonian energy functional (1), and that bounded energy, i.e., $H^{\varepsilon}(q, p)=\mathcal{O}(1)$ as $\varepsilon \rightarrow 0$, implies $g(q)=\mathcal{O}(\varepsilon)$. In other words, for $\varepsilon \ll 1$ solutions $q$ of bounded energy have to stay close to the constraint manifold

$$
\mathcal{M}=\left\{q \in \mathbb{R}^{N}:\|g(q)\|=0\right\} .
$$

From here on we will assume that $\operatorname{rank}(G(q))=L<N$ within the domain of interest, so that an explicit local decomposition of $q$ into fast and slow modes is possible according to the following

Remark 1.1. : Let $\Omega \subseteq \mathbb{R}^{N}$ be open and bounded and let $G(q) \in \mathbb{R}^{L \times N}$ with $\operatorname{rank} G(q)=L<N$ for all $q \in \Omega$, then the linear map $\mathcal{P}_{q}: \mathbb{R}^{N} \rightarrow \mathbb{R}^{N}$ given by

$$
\mathcal{P}_{q}:=G^{\mathrm{T}}(q)\left(G(q) G^{\mathrm{T}}(q)\right)^{-1} G(q)
$$

is an orthogonal projection.

Remark 1.2. : $\mathcal{P}_{q}^{\perp}$ denotes the orthogonal projection onto the orthogonal complement of the image of $\mathcal{P}_{q}$. For every $q \in \mathcal{M}$ its image is included in the corresponding tangent space to $\mathcal{M}$, in fact $\mathcal{P}_{q}^{\perp} \mathbb{R}^{N}=T_{q} \mathcal{M}$.

Remark 1.3. : As presented in [4] we can decompose the Hamiltonian energy functional in (11) into fast, slow and coupling energies after introducing a local coordinate transform into slow and fast variables (c.f. Lemma (2.6) below). The "fast" part of the Hamiltonian describes rapid oscillations in the fast variables orthogonal to the slow manifold $\mathcal{M}$ and depends on the slow variables, i.e., on the current nearest point on $\mathcal{M}$, only parametrically. In the original coordinates this fast or "oscillatory" part of the Hamiltonian reads

$$
H_{\mathrm{osc}}^{\varepsilon}(q, p)=\frac{1}{2} p^{\mathrm{T}} \mathcal{P}_{q} p+\frac{1}{2 \varepsilon^{2}} g(q)^{\mathrm{T}} K g(q)
$$

The above-mentioned coupling terms vanish for $\varepsilon \rightarrow 0$ in the present scenario of small amplitude oscillations, for diagonal positive definite $K$, and for eigenfrequencies of the individual components of the fast oscillations that are independent of the position on the manifold, see [46, 9]. As a consequence, the trajectories given by the full 
Hamiltonian in (11) follow the evolution determined by (5) closely for short times.

Henceforth we will assume $g$ to be locally smooth and $G(q)$ to have full $\operatorname{rank} L$ for all $q \in \mathbb{R}^{N}$ satisfying $\|g(q)\| \leq C$ for sufficiently large constant $C>0$. To state the setting more rigorously, we consider solutions $q^{\varepsilon}, p^{\varepsilon} \in C^{1}\left([0, T], \mathbb{R}^{N}\right)$ to (2) given the initial conditions

$$
\begin{array}{ll}
q^{\varepsilon}(0)=q_{0}^{0}+\varepsilon \bar{q}, & \bar{q} \in \mathbb{R}^{N}, \quad\left(q_{0}^{0}, p_{0}^{0}\right) \in \mathcal{T} \mathcal{M}, \\
p^{\varepsilon}(0)=p_{0}^{0}+\varepsilon \bar{p}, & \bar{p} \in \mathbb{R}^{N}
\end{array}
$$

where $\mathcal{T} \mathcal{M}$ denotes the tangential bundle of $\mathcal{M}$ which we will interpret as a manifold in phase space, i.e.,

$$
\mathcal{T} \mathcal{M}=\left\{(q, p) \in \mathbb{R}^{2 N}: q \in \mathcal{M} \wedge p \in T_{q} \mathcal{M}\right\}
$$

Therefore the initial data is, up to a perturbation of order $\varepsilon$, tangential [46], and we note in passing that the oscillatory energy (5) is of order $\mathcal{O}(1)$ in this case. Rubin and Ungar [46] proved existence of a unique solution $q^{0}, p^{0}$ to the differential algebraic equation system

$$
\begin{aligned}
\dot{q}^{0} & =p^{0} & & q^{0}(0)=q_{0}^{0} \in \mathcal{M} \\
\dot{p}^{0} & =-G^{\mathrm{T}}\left(q^{0}\right) K \lambda-\nabla V\left(q^{0}\right) & & p^{0}(0)=p_{0}^{0} \in T_{q_{0}^{0}} \mathcal{M} \\
g\left(q^{0}\right) & =0 & &
\end{aligned}
$$

and convergence $q^{\varepsilon}(t) \stackrel{\epsilon \rightarrow 0}{\longrightarrow} q^{0}(t), p^{\varepsilon}(t) \stackrel{\epsilon \rightarrow 0}{\longrightarrow} p^{0}(t)$ uniformly for $t \in[0, T]$ in the "tangential case" $(\bar{q}=0, \bar{p}=0)$. They obtained a similar result for the non-tangential case when the eigenfrequencies of the fast oscillations normal to the constraint manifold are independent of position. This is the scenario we pursue in our computational examples below. See [9] for an alternative elegant rigorous analysis in the case of co-dimension 1. The Lagrange multiplier $\lambda \in C^{1}\left([0, T], \mathbb{R}^{L}\right)$ can the be determined algebraically for every $t \in[0, T]$ by

$$
0=\ddot{g}\left(q^{0}\right)=-G\left(q^{0}\right)\left[\nabla V\left(q^{0}\right)+G^{\mathrm{T}}\left(q^{0}\right) K \lambda\right]+\sum_{i=1}^{N} \sum_{j=1}^{N}\left(\left(p^{0}\right)^{\mathrm{T}} e_{i, j} p^{0}\right) \frac{\partial^{2} g\left(q^{0}\right)}{\partial q_{i} \partial q_{j}}
$$

where $e_{i}$ is the $i$-th cartesian unit vector of $\mathbb{R}^{N}$ and $e_{i, j}=e_{i} \otimes e_{j} \in \mathbb{R}^{N \times N}$. Therefore this differential algebraic system is of index 3 and we obtain the additional hidden constraint $G\left(q^{0}\right) p^{0}=0$ by differentiation of $g\left(q^{0}\right)=0$ with regard to the parameter. Under additional assumptions [5] could prove that solutions $\left(q^{\varepsilon}, p^{\varepsilon}\right)$, initially $\varepsilon$-close to $\left(q^{0}, p^{0}\right)$, stay $\varepsilon$-close for exponentially long times. 
Example 1.4. Consider a chain of $L$ mass points with positions $r_{i} \in \mathbb{R}^{D}$ and momenta $v_{i} \in \mathbb{R}^{D}$ for $i=1, \ldots, L$. The first point (denoted by subscript 0) is assumed to be fixed, all points have equal mass and are under the influence of a constant unidirectional force $a_{0} e_{D}$ with $a_{0} \in \mathbb{R}$ and $e_{D}$ the unit vector in the last cartesian direction in $\mathbb{R}^{D}$. (Here we think of the gravitational force.) All points are pairwise connected by $L$ (linear) elastic bonds, characterized by their force coefficients $K=\operatorname{diag}\left(k_{1}, \ldots k_{L}\right)$ and their equilibrium lengths $l_{i}>0$ for $i=1 \ldots L$. Observing $N=D L$, we can describe the evolution of this mechanical system by (2) if we collect the components of all positions and momenta in $q$ and $p$, respectively. By means of classical mechanics we then conclude

$$
\begin{aligned}
& g(q)=\left(\begin{array}{lllll}
\left\|r_{1}\right\|-l_{1} & \ldots & \left\|r_{i}-r_{i-1}\right\|-l_{i} & \cdots & \left\|r_{L}-r_{L-1}\right\|-l_{L}
\end{array}\right)^{\mathrm{T}} \\
& V(q)=a_{0} \sum_{i=1}^{L} e_{D}^{\mathrm{T}} r_{i}
\end{aligned}
$$

If not stated differently, we assume $D=2$. Note that this is a genuinely nonlinear model with at least two time

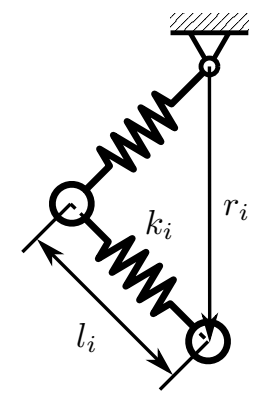

Figure 1: A graphical depiction of (2) using (10) for the case of two mass points $L=2$ moving in the plane $D=2$.

scales even for the simplest case of the elastic pendulum with $L=1$. Due to the work of [4], we expect solutions $q^{\epsilon}$ for this case to stay close to the solutions of the classical pendulum $q^{0}$ for long times. Since our goal is to observe and predict chaotic slow dynamics, we wish for a system with solutions $q^{0}$, which already exhibit chaotic behavior. For this reason we have to consider a slightly more complex model and choose $L=2$.

As the constraint manifold is of codimension $L=2$ the results of [46] are not applicable. Nevertheless one can, under additional assumptions, conclude convergence of the solution $\lim _{\varepsilon \rightarrow 0} q^{\epsilon}=q^{0}$ by the means of [48, 9]. For $L>1$ and non-resonant configurations [5] proved solutions $q^{\varepsilon}$ to stay close to $q^{0}$ for long times, if initially so.

Remark 1.5. As demonstrated in 43]

$$
g\left(q^{\varepsilon}\right)=\varepsilon^{2} \lambda\left(q^{\varepsilon}, p^{\varepsilon}\right)
$$

with $\lambda(q, p)$ determined by (9), is a better approximation to the slow dynamics of (2) than the zeroth order balance relation $(q, p) \in T \mathcal{M}$. Replacing the constraint in (8) by (11) leads to the concept of soft or flexible constraints as 
introduced in [43, 52$]$.

Remark 1.6. It should be noted that initial conditions with unconstrained momentum of the form

$$
\begin{aligned}
& q_{0}^{\varepsilon}=q_{0}^{0}+\varepsilon \tilde{q}_{0} \quad q_{0}^{0} \in \mathcal{M}, \tilde{q}_{0} \in \mathbb{R}^{N} \\
& p_{0}^{\varepsilon}=\tilde{p}_{0} \quad \tilde{p}_{0} \in \mathbb{R}^{N} \text {. }
\end{aligned}
$$

do not generally follow this theory. In this case, an additional force term can appear in the limiting equations (8) . See [46, 48, 9] for more details.

\subsection{Bayesian data assimilation}

When describing physical processes by models there are several sources of uncertainties, such as model errors or an uncertainty about the initial conditions. Ensemble-based data assimilation combines model outputs with, possibly also somewhat erroneous, observational data to estimate a probability distribution over model states conditioned on the observations. Owing to the usually high dimension of the model state space, probability densities are generally approximated by the empirical probability densities represented by ensembles of individual realizations of model runs.

Variational data assimilation seeks estimates of the trajectory of model states over an entire observation time interval by solving a related error minimization problem. A well known candidate of this kind is $4 \mathrm{D}-\mathrm{VAR}$, as explained e.g. in [45, p. 186].

An alternative is sequential Bayesian data assimilation. In a forecast step this method evolves the empirical probability distribution by forward simulation of the ensemble members until a new observation $y_{\text {obs }}$ becomes available. The resulting distribution is called the prior or forecast distribution, $\pi^{\mathrm{f}}$. In the analysis step, the observations are assimilated by applying Bayes' theorem to generate an improved posterior or analysis distribution, $\pi^{\mathrm{a}}$, that accounts for the observational evidence. This Bayesian step reads

$$
\pi^{\mathrm{a}}\left(z \mid y_{\mathrm{obs}}\right)=\frac{\pi\left(y_{\mathrm{obs}} \mid z\right)}{\pi^{f}\left(y_{\mathrm{obs}}\right)} \pi^{\mathrm{f}}(z)
$$

where $z=\left(q^{\mathrm{T}}, p^{\mathrm{T}}\right)^{\mathrm{T}} \in \mathbb{R}^{2 N}$ denotes model states.

In this work we focus on the second approach, and on how to apply sequential Bayesian data assimilation to models of the form (2). To this end we consider the deterministic evolution under these model equations given 
normally distributed initial data $z(0) \sim \mathcal{N}\left(z_{0}, Q\right)$ where $z_{0} \in T \mathcal{M}$. Furthermore we assume linear observations

$$
y_{\mathrm{obs}}\left(t_{k}\right)=H_{\mathrm{obs}} z\left(t_{k}\right)+\zeta
$$

where $H_{\text {obs }} \in \mathbb{R}^{I \times 2 N}$ is the matrix representing the linear observation map, and $\zeta \sim \mathcal{N}(0, R)$ is the measurement error with Gaussian statistics. Hereby $I \in \mathbb{N}$ is the dimension of the observation space.

For linear models, Gaussian measurement error, and Gaussian initial data, the Kalman filter solves the problem of optimally matching the analysis distributions to the observations [28]. Since the Gaussian structure of probabilities is exactly preserved in this case, the prior and posterior densities are completely characterized by their means $\bar{z}^{\mathrm{f}}\left(t_{k}\right), \bar{z}^{\mathrm{a}}\left(t_{k}\right)$ and covariances $P^{f}\left(t_{k}\right), P^{a}\left(t_{k}\right)$ at time $t_{k}$.

When the model equations are nonlinear and therefore the forecast distribution is not Gaussian anymore, we still can recover the main idea of the Kalman filter and approximate $\bar{z}^{\mathrm{f}}\left(t_{k}\right)$ and $P^{\mathrm{f}}\left(t_{k}\right)$ by their empirical counterpart and use the ensemble Kalman filter (EnKF) [17] to obtain the posterior mean $\bar{z}^{a}\left(t_{k}\right)$ and covariance $P^{a}\left(t_{k}\right)$. To be more specific we take the existing ensemble

$$
Z^{a}\left(t_{k-1}\right)=\left(z_{i}^{\mathrm{a}}\left(t_{k-1}\right)\right)_{i=1}^{M} \in \mathbb{R}^{2 N \times M}
$$

which represents the distribution $\pi^{a}\left(z, t_{k-1}\right)$ and evolve each member according to the model equations in time until $t_{k}$. Now the resulting ensemble $Z^{f}\left(t_{k}\right)=\left(z_{i}^{\mathrm{f}}\left(t_{k}\right)\right)_{i=1}^{M}$ samples the prior density $\pi^{f}\left(z, t_{k}\right)$ and we use $\bar{z}^{\mathrm{f}}\left(t_{k}\right) \approx$ $\frac{1}{M} \sum_{i=1}^{M} z_{i}^{\mathrm{f}}\left(t_{k}\right)=: \bar{z}^{\mathrm{f}}$ and $P^{\mathrm{f}}\left(t_{k}\right) \approx \frac{1}{M-1} \sum_{i=1}^{M}\left(z_{i}^{\mathrm{f}}\left(t_{k}\right)-\bar{z}^{\mathrm{f}}\right)\left(z_{i}^{\mathrm{f}}\left(t_{k}\right)-\bar{z}^{\mathrm{f}}\right)^{\mathrm{T}}$ to estimate the first and second moments of $\pi^{f}\left(z, t_{k}\right)$. To finally transform the prior samples to samples of the posterior, we assume a linear transformation

$$
z_{j}^{\mathrm{a}}\left(t_{k}\right)=\sum_{i=1}^{M} z_{i}^{\mathrm{f}}\left(t_{k}\right) \sigma_{i j}\left(t_{k}\right) \quad j=1, \ldots, M
$$

but are still left with a choice of the transformation matrix coefficients $\sigma_{i j}$ [45]. Our choice in this study will be the ensemble square root filter (ESRF) as described e.g. in [45, p.211-212]. The corresponding transfer matrix reads

$$
\sigma=\operatorname{diag}\left(w-\frac{1}{M}\right)+S
$$


where

$$
\begin{aligned}
& S=\left(I+\frac{1}{M-1}\left(H_{o b s} A\right)^{\mathrm{T}} R^{-1} H_{o b s} A\right)^{-\frac{1}{2}} \in \mathbb{R}^{M \times M}, \\
& w=\frac{1}{M} \sum_{i=1}^{M} e_{i}-\frac{1}{M-1} S^{2} A^{\mathrm{T}} H_{o b s}^{\mathrm{T}} R^{-1}\left(H_{o b s} \bar{z}^{\mathrm{f}}-y_{\mathrm{obs}}\right) \in \mathbb{R}^{M},
\end{aligned}
$$

with $e_{i} \in \mathbb{R}^{M},\left(e_{i}\right)_{j}=\delta_{i j}$, and where the "ensemble anomalies" are

$$
A=\left[z_{1}^{\mathrm{f}}\left(t_{k}\right)-\bar{z}^{\mathrm{f}}\left(t_{k}\right) \quad \ldots \quad z_{M}^{\mathrm{f}}\left(t_{k}\right)-\bar{z}^{\mathrm{f}}\left(t_{k}\right)\right] \in \mathbb{R}^{2 N \times M} .
$$

Using the ensemble square root filter we avoid the perturbation of the observations as necessary for non deterministic versions of the EnKF [51]. Nevertheless our statements do not depend on the specific choice made here.

\subsubsection{Failure of the plain ensemble square root filter}

Although the Hamiltonian (1) is conserved under the model dynamics (2), i.e.,

$$
H^{\varepsilon}\left(z_{i}^{\varepsilon \mathrm{f}}\left(t_{k+1}\right)\right)=H^{\varepsilon}\left(z_{i}^{\varepsilon \mathrm{a}}\left(t_{k}\right)\right)
$$

it is not conserved under transformation (16) which implements the data assimilation step. In particular, one often observes a severe increase in the oscillatory energy (5), i.e.

$$
H_{\mathrm{osc}}^{\varepsilon}\left(z_{i}^{\varepsilon \mathrm{a}}\left(t_{k}\right)\right) \gg H_{\mathrm{osc}}^{\varepsilon}\left(z_{i}^{\varepsilon \mathrm{f}}\left(t_{k}\right)\right)
$$

which, in practice, can lead to a destabilization of the simulation after a few data assimilation cycles. The reader is referred to [26, 30] for rigorous analyses of such possible catastrophic filter divergences. An explicit example of this effect is also provided below, see Fig. 2. For linear scalar balance relations, the situation can be controlled however, as summarized in the following

Remark 1.7. Let $\sigma \in \mathbb{R}^{M \times M}$ be the transformation matrix of a linear ensemble transform filter. Let furthermore $g(q)=G q$ with $G \in \mathbb{R}^{L \times N}$ a constant positive semi-definite matrix, so that $g$ is linear. Then for every ensemble of prior samples $q_{j}^{\mathrm{f}} \in \mathbb{R}^{N}$ and posterior samples $q_{j}^{\mathrm{a}} \in \mathbb{R}^{N}$ with $j \in\{1 \ldots M\}$

$$
g\left(q_{j}^{\mathrm{a}}\left(t_{k}\right)\right) \leq C \max _{i=1 \ldots m} g\left(q_{i}^{\mathrm{f}}\left(t_{k}\right)\right)
$$


at every time point $t_{k}$ with $C=\sum_{i=1}^{m}\left|\sigma_{i j}\right|$. In fact, due to the linearity of $g$ we can immediately conclude

$$
g\left(q_{j}^{\mathrm{a}}\right)=g\left(\sum_{i=1}^{m} q_{i}^{\mathrm{f}} \sigma_{i j}\right)=\sum_{i=1}^{m} g\left(q_{i}^{\mathrm{f}} \sigma_{i j}\right)=\sum_{i=1}^{m} \sigma_{i j} g\left(q_{i}^{\mathrm{f}}\right) \leq \sum_{i=1}^{m}\left|\sigma_{i j}\right| \max _{l=1 \ldots m} g\left(q_{l}^{\mathrm{f}}\right) .
$$

Remark 1.8. If the ensemble of prior samples is exactly balanced, i.e., satisfies $g\left(q_{i}^{\mathrm{f}}\right)=0$ for every $i=1 \ldots M$ then the ensemble of posterior samples will satisfy $g\left(q_{i}^{\mathrm{f}}\right)=0$ for every $i=1 \ldots M$, too.

Note that for nonlinear $g$ such control is not available, since neither (22) nor Remark 1.8 remain valid in general.

Although not of immediate importance for the current assimilation cycle, the assimilation reduces the mean distance of the ensemble to the observations as expected, and yet the subsequent forecast can be drastically wrong nevertheless. In the case of rather small $\varepsilon$ this can ultimately lead to filter divergence. An example of this situation is illustrated in Figure2, which also shows results with the improved balanced DA procedure to be described shortly.

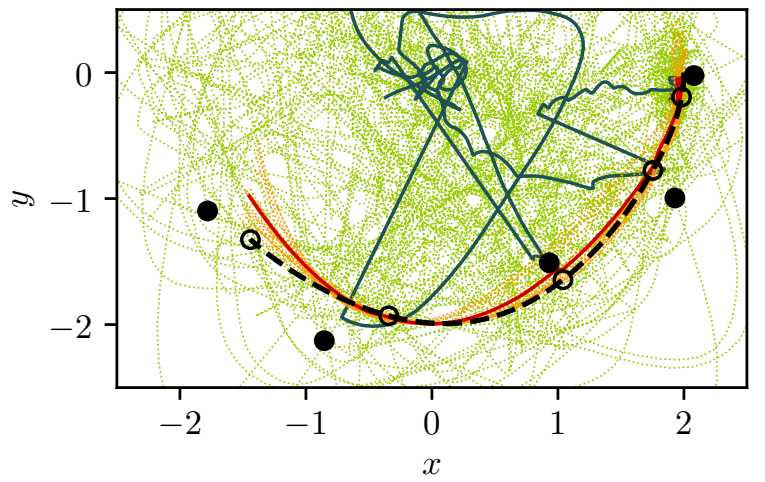

$$
\text { ens. ESRF }
$$$$
\text { est. ESRF }
$$

ens. bESRF

est. bESRF

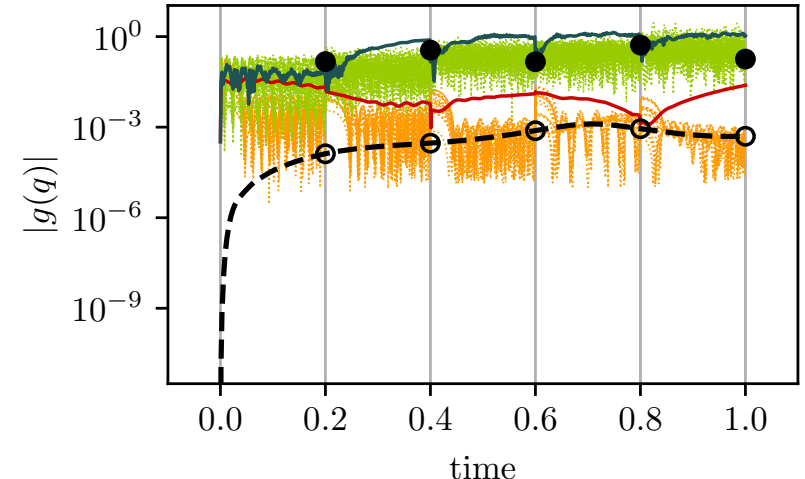

$\boldsymbol{- \theta}$ - reference

- observations

Figure 2: The divergence of an ensemble square root filter (ESRF) applied to the elastic stiff double pendulum as described in Example 1.4. The initial state was chosen as $q_{0}=(1,0,2,0)^{\mathrm{T}}, p_{0}=(0,0,0,0)^{\mathrm{T}}$ and the model parameters according to Table 1. The left figure depicts the ensemble trajectories (ens.) of the second mass point and the associated ensemble means (est.). The plain ESRF diverges after a couple of assimilation cycles, whereas the balanced version (bESRF) performs qualitatively well. The right figure shows the residual $\|g(q)\|$. The residual of the reference solution is non-zero as expected, but stays small. The residual of the balanced ESRF is drastically lower than that of the plain ESRF. We obtain these results using the method proposed in (67) below and the setup as described in Chapter [3 c.f. Table 1. In contrast to the situation there, we increase the initial spread and choose $\rho_{0}=0.1$. 
Balanced data assimilation

\section{Proposed methods}

To overcome the above-mentioned issue, we propose two different methods. The first, subsequently called "penalty method", observes and corrects the balance residuals after the assimilation algorithm. For this purpose we solve a minimization problem structurally similar to the 3DVar method (see e.g. 294). The second, subsequently called "blended time stepping method", is an extension of ideas first formulated in [3]. This approach does not modify the assimilated states but leverages an intermediate model as part of the forecast step that drives the evolution towards balanced states.

\subsection{Ensemble based penalty method}

Let $\hat{z}_{i}=\left(\hat{q}_{i}^{T}, \hat{p}_{i}^{T}\right)^{T}$ denote the coordinates and momenta of an ensemble $\hat{Z}$ provided by applying a linear ensemble transform filter to the forecasts $Z^{f}=\left(z_{i}^{f}\right)_{i=1}^{M}$. The $\hat{z}_{i}$ would represent the analysis ensemble if we were to ignore further balancing requirements. To improve the balance of these states, and to obtain the final analysis ensemble $Z^{a}$, we first generate an ensemble of updated imbalances by applying the ensemble Kalman filter transformation (16) to the forecasted values of $g$, i.e.,

$$
\hat{g}_{j}:=\sum_{i}^{M} g\left(q_{i}^{\mathrm{f}}\right) \sigma_{i j} \quad j \in\{1, \ldots, M\},
$$

and then minimize $L: \mathbb{R}^{N \times M} \rightarrow \mathbb{R}$, which acts on the ensemble of positions $Q=\left(q_{i}\right)_{i=1}^{M}$ only, and is defined through

$$
\begin{aligned}
L(Q) & =\frac{1}{2} \sum_{i=1}^{M}\left(\left(q_{i}-\hat{q}_{i}\right)^{\mathrm{T}} B\left(q_{i}-\hat{q}_{i}\right)+S_{i}\left(q_{i}\right)\right) \\
S_{i}\left(q_{i}\right) & =\left(g\left(q_{i}\right)-\gamma \hat{g}_{i}\right)^{\mathrm{T}} \Lambda\left(g\left(q_{i}\right)-\gamma \hat{g}_{i}\right)
\end{aligned}
$$

after each assimilation procedure in a post processing step. Note that ensemble members $i, j$ are not coupled in this minimization problem, so that it is equivalent to $M$ independent minimizations of smaller size.

The post-processed balanced posterior ensemble $Z^{\mathrm{a}}$ is now given as minimizers, i.e.,

$$
Z^{\mathrm{a}}=\left(\arg \min _{Q} L(Q), \hat{P}\right)
$$

where $\hat{P}$ denotes the ensemble of momenta from $\hat{Z}$.

In (25), $B \in \mathbb{R}^{N \times N}$ is a symmetric positive definite matrix and $\Lambda \in \mathbb{R}^{L \times L}$ is a positive definite diagonal matrix which weighs the importance of the proposed analysis ensemble against that of improved balance. The matrix $B$ is 
typically given by the inverse of the empirical covariance matrix of the ensemble $\hat{Q}$, i.e., the ensemble of coordinates

from $\hat{Z}$. The matrix $\Lambda$ is chosen to be diagonal with a common scaling factor $\ell>0$ which controls the impact of the balancing terms $S_{i}$ in (25a). Furthermore, the parameter $\gamma \in[0,1]$ controls the quality of balance to be achieved relative to the assimilated balance residuals $\hat{g}_{i}$. Whereas the $\hat{g}_{i}$ may already be reduced in amplitude relative to the forecast values $g\left(q_{i}^{f}\right)$ depending on the structure of the weights $\sigma_{i j}$, a stronger enforcement of balance in a single assimilation step may be advantageous. This is achieved by choosing values $\gamma<1$. The extreme case of $\gamma=0$, together with $\|B\| \ll\|\Lambda\|$ would amount to a projection of the forecasted states onto the balanced manifold, which - in turn - might not be desirable either as this may suppresses physically meaningful smaller imbalances, too. At this stage, the concrete choice of $\gamma$ remains subject to the particular application context. Additional physical considerations will provide further guidance towards a best practice in the choice of parameters.

Remark 2.1. We deliberately refrain from combining the assimilation algorithm and this post processing step into a single assimilation step for the sake of transparency and to advertise the flexibility of this post processing approach.

\subsubsection{Gauß-Newton minimization}

The gradient of (25a) evaluated at any minimizer thereof vanishes i.e.

$$
0=\frac{\partial L}{\partial q_{i}}(Q)=B\left(q_{i}-\hat{q}_{i}\right)+\nabla S_{i}\left(q_{i}\right) \quad \forall i \in\{1, \ldots, M\}
$$

For the approximate numerical solution of this system of coupled nonlinear equations we first summarize here the Gauss-Newton algorithm, which we modify slightly in the next section to obtain the method actually used in sample calculations below.

Let $Q^{k}$ denote the $k$ th iterate of the ensemble of positions. Then one linearizes the cost functional (25a) by

$$
g\left(q_{i}\right) \approx g\left(q_{i}^{k}\right)+G\left(q_{i}^{k}\right)\left(q_{i}-q_{i}^{k}\right)
$$

and thus obtains

$$
\left(B+G^{\mathrm{T}}\left(q_{i}^{k}\right) \Lambda G\left(q_{i}^{k}\right)\right)\left(q_{i}-q_{i}^{k}\right)=-B\left(q_{i}^{k}-\hat{q}_{i}\right)-\nabla S_{i}\left(q_{i}^{k}\right) \quad \forall i \in\{1, \ldots M\}
$$

as the linearized critical point condition. Solving this system of linear equations for the ensemble of increments 
$\Delta Q^{k}:=Q^{k+1}-Q^{k}$ is equivalent to the inversion of

$$
\tilde{B}_{i}^{k}:=\left(B+G\left(q_{i}^{k}\right)^{\mathrm{T}} \Lambda G\left(q_{i}^{k}\right)\right) .
$$

Each of these matrices is indeed invertible, since $B$ is symmetric positive definite and $\Lambda$ is strictly positive diagonal. This allows one to formulate the update increment for each ensemble member independently, i.e.,

$$
\Delta q_{i}^{k}=-\left(\tilde{B}_{i}^{k}\right)^{-1}\left(B\left(q_{i}^{k}-\hat{q}_{i}\right)+\nabla S_{i}\left(q_{i}^{k}\right)\right)
$$

Remark 2.2. For $L \ll N$ one may reduce the computational costs for each iteration step by expressing $\left(\tilde{B}_{i}^{k}\right)^{-1}=$ $\left(B+G\left(q_{i}^{k}\right)^{\mathrm{T}} \Lambda G\left(q_{i}^{k}\right)\right)^{-1}$ by the Sherman-Morrison-Woodbury formula [22, p. 51].

\subsubsection{Continuous formulation and a modified search direction}

Instead of using the gradient descent proposed by the Gauss-Newton method, we aim here to solve (27) using a different direction of descent. To this end we introduce a modified search direction, obtained by replacing $\Lambda$ in (30) by $\Lambda h$ where $h>0$. In analogy with (31) we denote the ensemble increment obtained by this method by $\Delta \tilde{Q}^{k}$, and we note that all fixed points of $Q^{k} \mapsto Q^{k}+h \Delta \tilde{Q}^{k}$ are solutions of (27) and therefore again candidates for minimizers.

A single iteration by this method turns out to be one step with pseudo-time increment $\Delta s=h$ of a stable numerical integrator for the auxiliary differential equation system

$$
\frac{d}{d s} q_{i}=-\left(q_{i}-\hat{q}_{i}\right)-B^{-1} G^{\mathrm{T}}\left(q_{i}\right) \Lambda\left(g\left(q_{i}\right)-\gamma \hat{g}_{i}\right) \quad \forall i \in\{1, \ldots, M\}
$$

This integrator, augmented with an adaptive step size optimized for rapid reduction of the residuum, and applied to the initial value problem with initial data $q_{i}(0)=\hat{q}_{i}$ constitutes the proposed minimizer for the balancing cost function in (25). This is also our preferred method used in the test cases below.

Remark 2.3. The evolution governed by (32) is a gradient flow driven by $L$ and the geometry of $\operatorname{diag}\left(B^{-1}, \ldots, B^{-1}\right) \in$ $\mathbb{R}^{M N \times M N}$. We therefore expect the solution of (32) to converge to an equilibrium solution $q_{\infty}=\lim _{t \rightarrow \infty} q(t)$ satisfying (27).

Proposition 2.4. The numerical method governed by (31) with $\Lambda$ replaced by $h \Lambda$ for a pseudo-time increment $s^{k+1}-s^{k}$, i.e.,

$$
q_{i}^{k+1}=q_{i}^{k}-h\left(B+h G^{\mathrm{T}}\left(q_{i}^{k}\right) \Lambda G\left(q_{i}^{k}\right)\right)^{-1}\left(B\left(q_{i}^{k}-\hat{q}_{i}\right)+\nabla S_{i}\left(q_{i}^{k}\right)\right)
$$


is consistent with (32). For $h \in(0,1)$ there exists $\delta>0$ such that every sequence $\left(q_{i}^{k}\right)_{k \in \mathbb{N}}$ determined by (33) and starting in the open ball $B_{\delta}\left(q_{i}^{\infty}\right)$ around an equilibrium solution $q_{i}^{\infty}$ converges to that solution.

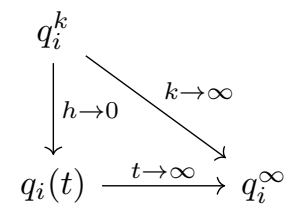

Figure 3: The commuting diagram shows the stability property of discretization (33).

Proof. We recall that $B \in \mathbb{R}^{N \times N}$ is invertible and bounded as it is finite dimensional. The expansion given by the Neumann series gives

$$
\begin{aligned}
\left(B+h G^{\mathrm{T}}\left(q_{i}^{k}\right) \Lambda G\left(q_{i}^{k}\right)\right)^{-1} & =\left(\mathbb{1}+h B^{-1} G^{\mathrm{T}}\left(q_{i}^{k}\right) \Lambda G\left(q_{i}^{k}\right)\right)^{-1} B^{-1}=\sum_{k=0}^{\infty}\left(-B^{-1} G^{\mathrm{T}}\left(q_{i}^{k}\right) \Lambda G\left(q_{i}^{k}\right)\right)^{k} B^{-1} \\
& =B^{-1}+\mathcal{O}(h) .
\end{aligned}
$$

This allows us to conclude consistency by a standard Taylor argument, expanding the solution $q_{i}$ at $t^{k}$ and assuming $q_{i}^{n}=q_{i}\left(t^{k}\right)$. For this purpose first rewrite

$$
\begin{aligned}
q_{i}^{k+1} & =q_{i}^{k}-h\left(B+h G^{\mathrm{T}}\left(q_{i}^{k}\right) \Lambda G\left(q_{i}^{k}\right)\right)^{-1} \frac{\partial L}{\partial q_{i}}\left(Q^{k}\right) \\
& =q_{i}^{k}-h B^{-1} \frac{\partial L}{\partial q_{i}}\left(Q^{k}\right)+\mathcal{O}\left(h^{2}\right)
\end{aligned}
$$

and subsequently conclude

$$
\left\|q_{i}\left(t^{k+1}\right)-q_{i}^{k+1}\right\|=\left\|q_{i}\left(t^{k}\right)-h B^{-1} \frac{\partial L}{\partial q_{i}}\left(Q\left(t^{k}\right)\right)-q_{i}^{k}+h B^{-1} \frac{\partial L}{\partial q_{i}}\left(Q^{k}\right)+\mathcal{O}\left(h^{2}\right)\right\|=\left\|O\left(h^{2}\right)\right\| .
$$

This implies global first order consistency and the first part of the statement. For the second part let $h \in(0,1)$. Subtracting $q_{i}^{\infty}$ on both sides and furthermore using

$$
0=B\left(q_{i}^{\infty}-\hat{q}_{i}\right)+G\left(q_{i}^{k}\right) \Lambda\left(g\left(q_{i}^{k}\right)-\overline{\hat{g}}\right)
$$

allows us to conclude equivalence of (33) and the following identity,

$$
\begin{aligned}
q_{i}^{k+1}-q_{i}^{\infty} & =q_{i}^{k}-q_{i}^{\infty}-h\left(B+h G^{\mathrm{T}}\left(q_{i}^{k}\right) \Lambda G\left(q_{i}^{k}\right)\right)^{-1}\left(B\left(q_{i}^{k}-q_{i}^{\infty}\right)+G^{\mathrm{T}}\left(q_{i}^{k}\right) \Lambda\left(g\left(q_{i}^{k}\right)-g\left(q_{i}^{\infty}\right)\right)\right) \\
& =\left(\mathbb{1}-h\left(B+h G^{\mathrm{T}}\left(q_{i}^{k}\right) \Lambda G\left(q_{i}^{k}\right)\right)^{-1}\left(B+G^{\mathrm{T}}\left(q_{i}^{k}\right) \Lambda G\left(q_{i}^{k}\right)\right)\right)\left(q_{i}^{k}-q_{i}^{\infty}\right)+h r\left(q_{i}^{k}, q_{i}^{k}-q_{i}^{\infty}\right)
\end{aligned}
$$


The last equality is valid as long as $q^{k} \in B_{\rho}\left(q^{\infty}\right)$ for sufficiently small $\rho>0$. In this case we can apply Taylor expansion which also gives us

$$
R\left(w_{i}\right):=\sup _{v \in B_{\rho}\left(q^{\infty}\right)}\left\|r\left(v_{i}, w_{i}\right)\right\| \in \mathcal{O}\left(\left\|w_{i}\right\|^{2}\right)
$$

The fact that $B$ and $G\left(q_{i}^{k}\right)^{\mathrm{T}} \Lambda G\left(q_{i}^{k}\right)$ are both symmetric positive definite allows us to conclude the following estimate

$$
\begin{aligned}
\left\|q_{i}^{k+1}-q_{i}^{\infty}\right\| & \leq\left\|\mathbb{1}-h\left(B+h G^{\mathrm{T}}\left(q_{i}^{k}\right) \Lambda G\left(q_{i}^{k}\right)\right)^{-1}\left(B+G^{\mathrm{T}}\left(q_{i}^{k}\right) \Lambda G\left(q_{i}^{k}\right)\right)\right\|\left\|q_{i}^{k}-q_{i}^{\infty}\right\|+h R\left(q_{i}^{k}-q_{i}^{\infty}\right) \\
& \leq(1-h)\left\|\left(B+h G^{\mathrm{T}}\left(q_{i}^{k}\right) \Lambda G\left(q_{i}^{k}\right)\right)^{-1} B\right\|\left\|q_{i}^{k}-q_{i}^{\infty}\right\|+h R\left(q_{i}^{k}-q_{i}^{\infty}\right) \\
& \leq \frac{(1-h)\|B\|}{\left\|\left(B+h G^{\mathrm{T}}\left(q_{i}^{k}\right) \Lambda G\left(q_{i}^{k}\right)\right)\right\|}\left\|q_{i}^{k}-q_{i}^{\infty}\right\|+h R\left(q_{i}^{k}-q_{i}^{\infty}\right) \\
& \leq(1-h)\left\|q_{i}^{k}-q_{i}^{\infty}\right\|+h C_{1}\left\|q_{i}^{k}-q_{i}^{\infty}\right\|^{2} \\
& \leq\left(1-h+h C_{1}\left\|q_{i}^{k}-q_{i}^{\infty}\right\|\right)\left\|q_{i}^{k}-q_{i}^{\infty}\right\| \\
& \leq C_{2}\left\|q_{i}^{k}-q_{i}^{\infty}\right\| .
\end{aligned}
$$

Here the constant satisfies $C_{1}<1$ as long as $q_{i}^{k}$ is already close enough to $q_{i}^{\infty}$. If so, then we immediately obtain $\left\|q_{i}^{k+1}-q_{i}^{\infty}\right\| C_{1}<1$ and therefore $C_{2}<1$ too. An inductive argument finally implies convergence to the equilibrium solution $q_{i}^{\infty}$ for sufficiently close initial value.

\section{$2.2 \quad$ Blended time-stepping}

Motivated by the results of [3], we introduce a numerical time stepping scheme that extends a classical projection approach by subsequent blending steps. These steps begin with a classical projection step and then access successively less constrained intermediate models along a one-parameter model family that continuously bridges between the unconstrained original model (2) and the reduced and its fully constrained limit (8). This approach was originally developed in the context of incompressible fluid dynamics where the singular perturbation arises by the vanishing Mach number limit $\mathrm{Ma} \rightarrow 0$. In addition to the classical projection schemes introduced in [12], much effort was spent on developing asymptotic preserving low Mach number numerical schemes and variants thereof, [see, e.g., 33, 15, 27]. The essential point is their ability to blend between the (weakly) compressible and the incompressible dynamics without additional stability constraints. As observed in [3], solving the incompressible model immediately after the assimilation for one or two time steps and subsequently blending smoothly back to the compressible model over another few time steps can further reduce artificial imbalances caused by data assimilation relative to an approach that simply projects the system state onto the incompressible manifold in one step and then proceeds with the compressible model. 
To adapt this strategy to our situation, we introduce the following family of blended models, controlled by the blending parameter $\alpha$,

$$
\begin{array}{ll}
\dot{q}=p, & q(0)=q_{0} \in \mathbb{R}^{N} \\
\dot{p}=-G^{\mathrm{T}}(q) K\left(\frac{\alpha}{\varepsilon^{2}} g(q)+(1-\alpha) \lambda^{0}\right)-\nabla V(q), & p(0)=p_{0} \in \mathbb{R}^{N}
\end{array}
$$

where $\lambda^{0}$ is the lagrangian multiplier as calculated from the limit problem

$$
\begin{aligned}
\dot{q} & =p, & q(0) & =q_{0} \in \mathbb{R}^{N} \\
\dot{p} & =-G^{\mathrm{T}}(q) K \lambda^{0}-\nabla V(q), & & p(0)=p_{0} \in \mathbb{R}^{N} \\
G(q) p & =0 . & &
\end{aligned}
$$

Obviously, for $\alpha=1$ we recover the unconstrained dynamics from (2), whereas for $\alpha=0$ and provided $\left(q_{0}, p_{0}\right) \in T \mathcal{M}$, we recover the constrained dynamics from (8). Note, however, that in this blended model we consciously use the hidden limit constraint for the momenta, i.e., $G(q) p=0$, rather than the original constraint $g(q)=0$. As we will demonstrate below, this ensures a desired dissipative behavior towards balanced solutions when $\varepsilon$ is small but non-zero and $0<\alpha<1$. For the limiting cases $\alpha \in\{0,1\}$, however, the non-dissipative symplectic integrator is maintained.

Let us denote by

$$
z^{n+1}=\psi_{h}^{\alpha}\left(z^{n}\right) \quad \alpha \in[0,1]
$$

a numerical discretization of (41), so that the operator $\psi_{h}^{\alpha}$ advances a given solution $z^{n}$ at time $t^{n}$ by a time step $h=t^{n+1}-t^{n}$ to the next time level. For $\alpha=0$ we employ a projection method that keeps the momenta tangential to the manifold $g(q)=g\left(q_{0}\right)$, whereas for $\alpha=1$ we use the nearly energy-preserving Stömer-Verlet scheme to resolve the unconstrained model (2). The time discretization for $\alpha \in(0,1)$ will have dissipative character as shown in sections 2.2.1, 2.2.2 below, and it is designed to efficiently remove any artificially introduced oscillatory energy from the system.

In this approach we accept a non-vanishing consistency error with respect to the fast model when evolving the system with $\alpha \in[0,1)$. But, as discussed in the beginning, we can assume the solutions of the unconstrained system to stay $\varepsilon$-close to the solutions of the constrained one. This enables us to locally decompose the consistency error into two parts, one in $\mathcal{M}$, caused by the nonlinearity of $V$ and another one orthogonal to $\mathcal{M}$. The slow first part is assumed to be captured by the data assimilation, whereas the second fast part is small of order $\mathcal{O}(\varepsilon)$ as 
discussed before. Yet, when artificial imbalances are introduced through a data assimilation procedure, the latter assumption ceases to be valid. In this case, the discrete evolution of the blended method should rapidly dampen the fast oscillations orthogonal to $\mathcal{M}$ as long as $\alpha \in(0,1)$ and until they attain the correct magnitude of $\mathcal{O}(\varepsilon)$. To this end we propose to use the blending method (42) as follows. Let us denote the blending window by $k \geq 1$ and start our forecast at time $t_{n}$. Let $\eta$ be the number of forecast time integration steps. Then the following two steps are repeated in every forecast cycle (c.f. Figure 4).

1. Blending: Let $\alpha \in \mathbb{R}^{k}$ such that $0=\alpha_{1}<\alpha_{2} \leq \cdots \leq \alpha_{k-1}<\alpha_{k}=1$. Integrate until $t_{n+k}$ using

$$
q_{n+k}=\left(\psi_{h}^{\alpha_{k}} \circ \psi_{h}^{\alpha_{k-1}} \circ \cdots \circ \psi_{h}^{\alpha_{1}}\right)\left(q_{n}\right)
$$

2. Forecast: Obtain forecast at $t_{n+\eta}$ by evolving $q_{n+k}$ along $\psi_{h}^{1}$ for $\eta-k$ - time steps.

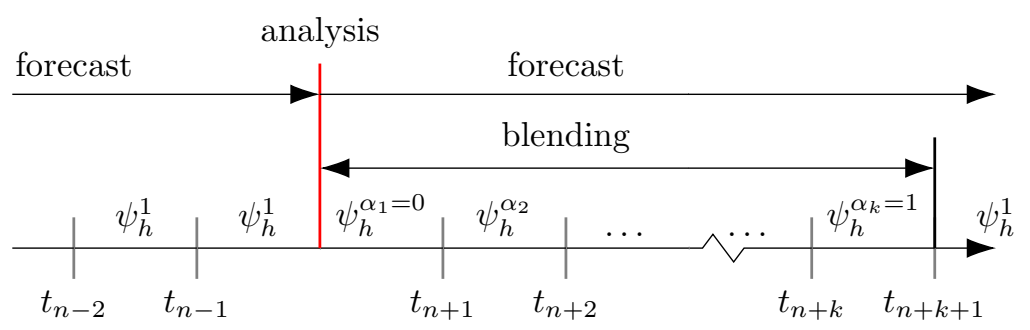

Figure 4: Blended time stepping applied after analysis with blending window $k$. The numerical flow $\psi_{h}^{\alpha}$ with step width $h$ is given by (42).

Figure 5 illustrates the qualitative behaviour of the blended time stepping for the stiff elastic double pendulum, introduced in Example 1.4, with slightly unbalanced initial coordinates. We observe that with respect to the balance residual the blended time stepping improves the situation drastically. After short time the residuals of the initially unbalanced and initially balanced solution match. Since we dissipate energy in the fast variables (c.f. Lemma 2.7) as long as $\alpha \notin\{0,1\}$, the overall energy of the system decreases as expected and the slow rotational motion of the stiff double pendulum is therefore resolved reasonably well with regard to balance and energy. Nevertheless, due to the lack of a priori knowledge so far, we chose $\alpha \in[0,1]$ and as a linear function of time, but as we already can guess from the form of the decay, this is a brute force and suboptimal choice in the sense that we can find a smaller range of $\alpha$ within which the solution relaxes to the slow motion more quickly. We leave the development of an optimized control of the blending sequence for future work. 

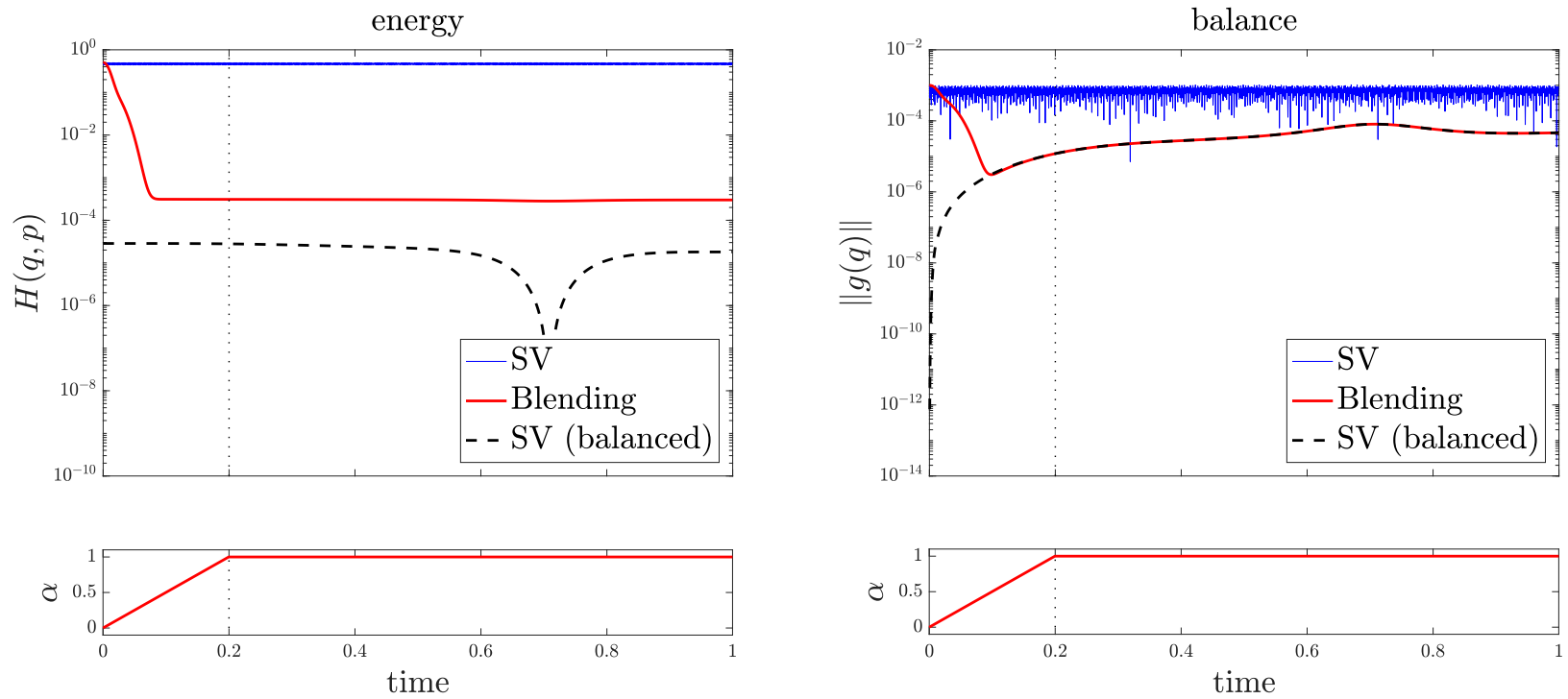

Figure 5: Energy and residual of balance relation for the stiff elastic double pendulum (c.f. Example 1.4 and Fig. 2). Initially unbalanced numerical solutions based on the Störmer-Verlet (SV) (blue) and the blending method (red), respectively. The corresponding parameter $\alpha$ for the blended time stepping is shown and the start of the pure forecasting region is marked by a vertical dotted line. As the reference we display the data for an initially balanced solution, computed again by the Störmer-Verlet method (dashed).

\subsubsection{A dissipative model family}

The aim of the present and the next sections is to analyse the proposed balanced data assimilation strategy, and in particular its behavior within the blending window while $\alpha \in(0,1)$, in the spirit of a "modified equation" analysis [50]. The modified equation system that effectively represents our discretization of the blended model reads

$$
\begin{aligned}
& \dot{\tilde{q}}=\tilde{p} \\
& \dot{\tilde{p}}=-\frac{1}{\varepsilon^{2}} G(\tilde{q})^{\mathrm{T}} K g(\tilde{q})-d \mathcal{P}_{\tilde{q}} \tilde{p}-\nabla V(\tilde{q}),
\end{aligned}
$$

where $d$ is a scalar damping coefficient. A key characteristic of this model is that, owing to the projection $\mathcal{P}_{\tilde{q}}$ in the damping term (see (4)), the dissipation term acts predominantly on the fast oscillatory components of the solution, thereby pulling initially unbalanced states towards balanced conditions as time evolves. In section 2.2 .2 we will show that a particular time discretization of the blended model in (41) is consistent with solutions of this dissipative surrogate model when the relationship $\alpha=(1-h d)$ between the blending parameter $\alpha$ from (41), the damping parameter $d$ from (44), and the time step size $h$ is observed. This analogy, in the sense of a modified equation analysis of the discretization, will largely explain the success of the proposed blending strategy in controlling unwanted imbalances in a data assimilation procedure. See also Fig. 6 below. Note that a similar damping term 
was first proposed in the context of numerical stabilization techniques in [20].

Since fast and slow energy parts of the Hamiltonian (1) can be separated only by an asymptotic argument and are coupled nonlinearly, we do not expect the surrogate system in (44) to completely dissipate the fast energy of (10). Nevertheless, in reasonably well separated cases the impacts of finite $\varepsilon$ and nonlinearity will be negligible, especially in the context of data assimilation, where the correct slow energy itself is anyway known only with limited accuracy.

To develop an intuition regarding the behavior of solutions to (44) we discuss the arguably simplest model in the class of such problems with multiple scales, the uncoupled harmonic oscillator.

Example 2.5 (Damped harmonic oscillator). Let $q=(\mu, \nu)^{\mathrm{T}}, p=(\eta, \zeta)^{\mathrm{T}}, K=\operatorname{diag}\left(\varepsilon^{2}, 1\right)$ and $V(q)=0$

$$
\begin{array}{ll}
\dot{\mu}=\eta & \dot{\eta}=-\mu \\
\dot{\nu}=\zeta & \dot{\zeta}=-\frac{1}{\varepsilon^{2}} \nu-4 d \zeta
\end{array}
$$

The well known analytical solutions for the damped harmonic oscillator are given by $\mu=\mu_{0} \cos (t)+\nu_{0} \sin (t)$ and

$$
\nu= \begin{cases}e^{-2 d t}\left(\nu_{0}+t\left(2 d \nu_{0}+\zeta_{0}\right)\right) & 2 d=\frac{1}{\varepsilon} \\ e^{-2 d t}\left(\left(\nu_{0}+\frac{\zeta_{0}}{\omega_{d}}\right) e^{i \omega_{d} t}+\left(\nu_{0}-\frac{\zeta_{0}}{\omega_{d}}\right) e^{-i \omega_{d} t}\right) & 2 d \neq \frac{1}{\varepsilon}\end{cases}
$$

where the frequency for the fast damped component is given by $\omega_{d}=\sqrt{\frac{1}{\varepsilon^{2}}-4 d^{2}}$. We immediately realize that $d=0$ gives us the solution for the highly oscillatory system (2) and furthermore relaxes to the constraint (in this case also slow) manifold exponentially. In the general nonlinear and coupled case we present the corresponding result in Lemma 2.7 below.

For the overdamped limit, i.e., for $d \varepsilon \rightarrow \infty$ as $\varepsilon \rightarrow 0$ and $d \rightarrow \infty$, we conclude uniform convergence to the same solution $(\mu, 0)$ as for the constrained system as long as $\nu_{0} \in o(1 / d)$ and $\zeta_{0} \in o(1)$. Again this result can be stated in more general form and is presented in Lemma 2.9 .

The following lemma summarizes the well known (c.f. [44]) split of variables into a slow tangential and a fast normal part. It will enable us to identify slow and fast variables with respect to the different asymptotic limits.

Lemma 2.6. Any solution $(q, p)$ of System (44) can be split into components $\mu, \eta$ and $\nu, \zeta$ which satisfy

$$
\begin{aligned}
& q=E^{\mathrm{T}} \mu+G^{\mathrm{T}}\left(G G^{\mathrm{T}}\right)^{-\frac{1}{2}} \nu \\
& p=E^{\mathrm{T}} \eta+G^{\mathrm{T}}\left(G G^{\mathrm{T}}\right)^{-\frac{1}{2}} \zeta
\end{aligned}
$$


In the new coordinates $((\mu, \eta),(\nu, \zeta))$, system (44) is equivalent to

$$
\begin{aligned}
& \dot{\mu}=\dot{E} q+\eta \quad \dot{\eta}=\dot{E} p-E \nabla V(q) \\
& \dot{\nu}=\left(\frac{d}{d t}\left(G G^{\mathrm{T}}\right)^{-\frac{1}{2}} G\right) p+\zeta \quad \dot{\zeta}=\left(\frac{d}{d t}\left(G G^{\mathrm{T}}\right)^{-\frac{1}{2}} G\right) p-\varepsilon^{-2}\left(G G^{\mathrm{T}}\right)^{\frac{1}{2}} K g(q)-d \zeta-\left(G G^{\mathrm{T}}\right)^{-\frac{1}{2}} G \nabla V(q) .
\end{aligned}
$$

Furthermore the fast energy $H_{\text {osc }}$ satisfies

$$
H_{o s c}^{\varepsilon}((\nu, \zeta),(\mu, \eta))=\frac{1}{2} \zeta^{\mathrm{T}} \zeta+\frac{1}{2 \varepsilon^{2}} g(q(\mu, \nu))^{\mathrm{T}} K g(q(\mu, \nu))
$$

Proof. For the sake of readability we omitted the argument $q$ for $G, E$ in the statement of the Lemma and will do so throughout the proof. We split momenta tangential and orthogonal to $\mathcal{M}$ denoted by $\eta$ and $\zeta$ as well as the coordinates denoted by $\mu$ and $\nu$, respectively. More concretely we choose

$$
\begin{array}{ll}
\mu=E q & \eta=E p \\
\nu=\left(G G^{\mathrm{T}}\right)^{-\frac{1}{2}} G q & \zeta=\left(G G^{\mathrm{T}}\right)^{-\frac{1}{2}} G p
\end{array}
$$

where the columns of $E \in \mathbb{R}^{(N-L) \times N}$ are an orthonormal basis of $\left(\mathcal{P}_{q} \mathbb{R}^{N}\right)^{\perp}$. It is easy to check that $E^{\mathrm{T}} E$ is a orthogonal projection onto $\left(\mathcal{P}_{q} \mathbb{R}^{N}\right)^{\perp}$ and since orthogonal projections onto a fixed subspace are unique (for every q), we already know $E^{\mathrm{T}} E=\mathcal{P}_{q}^{\perp}=\mathbb{1}-\mathcal{P}_{q}$. Substituting (50) into the right hand side of (47) we get

$$
E^{\mathrm{T}} E q+G^{\mathrm{T}}\left(G G^{\mathrm{T}}\right)^{-1} G q=\mathcal{P}_{q}^{\perp} q+\mathcal{P}_{q} q=q
$$

Since we used the same geometry to split the momenta this already implies (47).

We differentiate (50) and use system (44) for $\dot{q}$ and $\dot{p}$. Since by construction $G^{\mathrm{T}} E=0=E^{\mathrm{T}} G$ most of the terms drop and we conclude (48a) and (48b) after some straightforward algebraic manipulation. Recalling $G G^{\mathrm{T}}$ is symmetric positive definite, the last statement (49) finally follows from

$$
\begin{aligned}
H_{o s c}^{\varepsilon}(q, p) & =\frac{1}{2} p^{\mathrm{T}} \mathcal{P}_{q} p+\frac{1}{2 \varepsilon^{2}} g(q)^{\mathrm{T}} K g(q) \\
& =\frac{1}{2} p^{\mathrm{T}} G^{\mathrm{T}}\left(G G^{\mathrm{T}}\right)^{-\frac{1}{2}}\left(G G^{\mathrm{T}}\right)^{-\frac{1}{2}} G p+\frac{1}{2 \varepsilon^{2}} g(q)^{\mathrm{T}} K g(q) \\
& =\frac{1}{2} \zeta^{\mathrm{T}} \zeta+\frac{1}{2 \varepsilon^{2}} g(q)^{\mathrm{T}} K g(q) .
\end{aligned}
$$


Lemma 2.7. Let $d=\frac{c}{\varepsilon}$, with $c>0$ fixed. Then solutions to (44) which initially satisfy $H_{\text {osc }}^{\varepsilon}(q, p) \in \mathcal{O}\left(\varepsilon^{-2}\right)$, dissipate fast energy down to some residual of order $H_{o s c}^{\varepsilon} \in \mathcal{O}(1)$, if only $\varepsilon$ is sufficiently small.

Proof. Again we will omit the $\operatorname{arguments}$ of $E$ and $G$ for notational convenience. Additionally we introduce $\Gamma:=$ $\left(G G^{\mathrm{T}}\right)^{-\frac{1}{2}} G$. We will prove the statement by arguments from geometric singular perturbation theory [18]. For this purpose we split system (44) into slow and fast parts by the means of Lemma 2.6. Subsequently we multiply by $\varepsilon$ and rescale $\hat{\zeta}=\varepsilon \zeta$ which results in

$$
\begin{aligned}
\dot{\mu}=\dot{E} q+\eta & \dot{\eta}=\dot{E} p-E \nabla V(q) \\
\varepsilon \dot{\nu}=\varepsilon \dot{\Gamma} q+\hat{\zeta} & \dot{\hat{\zeta}}=\varepsilon^{2} \dot{\Gamma} p-\left(G G^{\mathrm{T}}\right)^{\frac{1}{2}} K g(q)-\varepsilon d \hat{\zeta}-\varepsilon^{2}\left(G G^{\mathrm{T}}\right)^{-\frac{1}{2}} G \nabla V(q) .
\end{aligned}
$$

We denote the right hand side of the fast variables by

$$
F((\nu, \hat{\zeta}),(\mu, \eta), \varepsilon(d))=\left(\begin{array}{c}
\varepsilon \dot{\Gamma}+\hat{\zeta} \\
-\varepsilon^{2} \dot{\Gamma} p-\left(G G^{\mathrm{T}}\right)^{\frac{1}{2}} K g(q)-\varepsilon d \hat{\zeta}-\varepsilon^{2}\left(G G^{\mathrm{T}}\right)^{-\frac{1}{2}} G \nabla V(q)
\end{array}\right)
$$

In the limit $d \varepsilon=$ const., $\varepsilon \rightarrow 0$ we identify the critical manifold as

$$
\mathcal{T} \hat{\mathcal{M}}:=\left\{(\eta, \hat{\zeta}, \nu, \mu) \in \mathbb{R}^{2 N}: g(q(\mu, \nu))=0 \wedge \hat{\zeta}=0\right\}
$$

Next we prove normal hyperbolicity of the critical manifold, i.e., we show that there are no eigenvalues of $\frac{\partial F}{\partial(\nu, \hat{\zeta})}$ with vanishing real part. The gradient evaluated on the manifold $\mathcal{T} \hat{\mathcal{M}}$ and for $\varepsilon=0$ is given by the block matrix

$$
D F:=\left.\frac{\partial}{\partial(\nu, \hat{\zeta})} F((\nu, \hat{\zeta}),(\mu, \eta), \varepsilon)\right|_{(\nu, \hat{\zeta}) \in \mathcal{T} \hat{\mathcal{M}}, \varepsilon=0}=\left(\begin{array}{cc}
0 & \mathbb{1} \\
-\left(G G^{\mathrm{T}}\right)^{\frac{1}{2}} K\left(G G^{\mathrm{T}}\right)^{\frac{1}{2}} & -\mathbb{1}
\end{array}\right)
$$

To compute the eigenvalues of this non symmetric matrix, we first recall that $\left(G G^{\mathrm{T}}\right)^{\frac{1}{2}}$ is symmetric positive definite and since $K$ is a strictly positive diagonal matrix, $\tilde{K}:=\left(G G^{\mathrm{T}}\right)^{\frac{1}{2}} K\left(G G^{\mathrm{T}}\right)^{\frac{1}{2}}$ is symmetric and positive definite, i.e., it has $L$ positive eigenvalues $\omega_{\tilde{K}, j}>0$. Therefore we conclude zero is no eigenvalue of $D F$ by $\operatorname{det} D F=\operatorname{det}(-\mathbb{1}) \operatorname{det}(-\tilde{K})$. Using the Schur complement again we argue for some eigenvalue $\omega \neq 0$ of $D F$

$$
\operatorname{det}(D F-\omega \mathbb{1})=\operatorname{det}(-\omega \mathbb{1}) \operatorname{det}\left(-(1+\omega) \mathbb{1}-\frac{1}{\omega} \tilde{K}\right) .
$$


The determinant vanishes if and only if there is $j \in\{1, \ldots, L\}$ such that

$$
-\omega(\omega+1)=\omega_{\tilde{K}, j}
$$

Solving this quadratic equation already provides us with all possible eigenvalues by

$$
\omega_{ \pm, j}=\frac{-1 \pm \sqrt{1-4 w_{\tilde{K}, j}}}{2}
$$

We directly observe $\operatorname{Re} \omega_{ \pm, j}<0$ for all $j \in\{1, \ldots L\}$ and therefore notice that $\hat{\mathcal{M}}$ is normally hyperbolic. By finally applying Fenichel's theorem we obtain existence of slow manifolds $S^{\varepsilon}$ (c.f. [34]) $\varepsilon$-close to a compact submanifold $S \subset \mathcal{T} \hat{\mathcal{M}}$ of the critical one as long as $\varepsilon$ is sufficiently small. More specifically we conclude for any $\left(\left(\eta^{\varepsilon}, \zeta^{\varepsilon}\right),\left(\mu^{\varepsilon}, \nu^{\varepsilon}\right)\right) \in S^{\varepsilon}$

$$
\begin{aligned}
g\left(q\left(\mu^{\varepsilon}, \nu^{\varepsilon}\right)\right)^{\mathrm{T}} K g\left(q\left(\mu^{\varepsilon}, \nu^{\varepsilon}\right)\right) & \leq c_{1} \varepsilon^{2} \\
\hat{\zeta}^{\varepsilon \mathrm{T}} \hat{\zeta}^{\varepsilon} & \leq c_{2} \varepsilon^{2}
\end{aligned}
$$

and therefore

$$
\max _{\left.\left(\eta^{\varepsilon}, \zeta^{\varepsilon}\right),\left(\mu^{\varepsilon}, \nu^{\varepsilon}\right)\right) \in S} H_{o s c}^{\varepsilon}\left(\left(\eta^{\varepsilon}, \zeta^{\varepsilon}\right),\left(\mu^{\varepsilon}, \nu^{\varepsilon}\right)\right) \in \mathcal{O}(1)
$$

Another consequence of Fenichel's theorem is that the dynamical behaviour of the linearization $D F$ of the fast subsystem on the critical manifold already determines the dynamical behaviour of solutions starting off a slow manifold $S^{\varepsilon}$. Since all eigenvalues of $D F$ have negative real part, we conclude $S$ as well $S^{\varepsilon}$ is attracting. Therefore any solution starting nearby will approach some $S^{\varepsilon}$ which finally implies the energy dissipation as stated.

Subsequently we will use (44) to establish a model hierarchy which resembles the analytical counterparts discretized by the blended numerical method (42). The following two lemmata concern the behaviour of the limit cases $d \rightarrow 0$ and $d \rightarrow \infty$. The first one is based on the classical result of continuous dependency on initial data and parameters for ordinary differential equations with continuously differentiable right hand side. In both cases we fix $\varepsilon>0$ and omit this standard proof.

Lemma 2.8. Let $\varepsilon>0$ be fixed. Solutions $(\tilde{q}, \tilde{p})$ of the dissipative system (44) approach solutions of the purely Hamiltonian system (2) as $d \rightarrow 0$.

For the other part $d \rightarrow \infty$ we use again geometric singular perturbation theory and we can conclude a slightly different type of statement in terms of invariant manifolds. 
Lemma 2.9. Let $d$ be sufficiently large and $\varepsilon^{2} \in o(1 / d)$. For every compact subset of $\mathcal{M}_{\infty}:=\left\{(q, p) \in \mathbb{R}^{2 N}\right.$ : $\zeta(q, p)=0\}$ (c.f. Lemma 2.6) there exists a manifold $\mathcal{M}_{1 / d}$ which lies within $\mathcal{O}(1 / d)$ of this subset and is locally invariant under the evolution of (44). Furthermore every solution starting sufficiently close to $\mathcal{M}_{1 / d}$ will approach $\mathcal{M}_{1 / d}$.

Proof. As pointed out we aim to apply geometric singular perturbation theory again. Therefore we start as before by splitting slow and fast momenta explicitly utilizing Lemma 2.6. Contrary to the situation in Lemma 2.7 the coordinates then are both slow variables. By dividing the momentum equation in (48b) by $d$ and passing to the limit $d \rightarrow \infty$ we obtain the critical manifold as $\mathcal{M}_{\infty}=\left\{(q, p) \in \mathbb{R}^{2 N}: \zeta(q, p)=0\right\}$. We denote the right hand side of the momentum equation in (48b) by $F((\eta, \zeta), q, 1 / d)$ and linearize on $\mathcal{M}_{\infty}$.

$$
D F:=\left.\frac{\partial}{\partial \zeta} F((\eta, \zeta), q, 1 / d)\right|_{(\nu, \hat{\zeta}) \in \mathcal{M}_{\infty}, \varepsilon=0}=-\left(G G^{\mathrm{T}}\right)
$$

Since $G G^{\mathrm{T}}$ is positive definite and by assumption $\operatorname{rank}\left(G G^{\mathrm{T}}\right)=L$ we conclude that $-\left(G G^{\mathrm{T}}\right)^{1 / 2}$ has exactly $L$ negative Eigenvalues. $M_{\infty}$ is therefore normally hyperbolic and we now infer by Fenichel's theorem [34] existence of an invariant (with respect to (44)) manifold $\mathcal{M}_{1 / d}, 1 / d$ - close to a compact subset of our choice of $\mathcal{M}_{\infty}$, exactly as stated. Since we additionally have only a stable subspace on $\mathcal{M}_{\infty}$ we gain the attractive behavior of $\mathcal{M}_{1 / d}$ by the same theorem.

Remark 2.10. The same statement is true if we take a compact submanifold of $\mathcal{M}_{\infty}$ and therefore also cover the case where the evolution starts on the constraint manifold $\mathcal{M} \subseteq \mathcal{M}_{\infty}$.

Corollary 2.11. Let $d$ be sufficiently large and $\varepsilon^{2} \in o(1 / d)$. For every compact subset of the constraint manifold $\mathcal{M}$ there exists a manifold $\mathcal{M}_{1 / d}$ which lies within $\mathcal{O}(1 / d)$ of this subset and is locally invariant under the evolution of (44). Furthermore every solution starting sufficiently close to $\mathcal{M}_{1 / d}$ will approach $\mathcal{M}_{1 / d}$.

Remark 2.12. Although the preceding corollary tells us there is at least one slow manifold for large $d$ that satisfies the constraint, this does not imply we approach one of this kind, when starting slightly off the original constraint manifold $\mathcal{M}$.

So far we have only considered the analytical properties of the dissipative system (44). Building upon the insights gained, we now propose a related numerical method. 


\subsubsection{Dissipative discretization of the intermediate blended models}

Here we will establish a relation between discrete solutions to the blended model from (41) and solutions of the dissipative surrogate model from (44) in the spirit of a modified equation analysis [50]. In other words, we will argue that trajectories produced by the blended method will locally relax to the constraint manifold by similar means as in the context of the numerical stabilization of solvers for differential algebraic equations [2]. It will be useful to consider the following generalization of the constrained system (8), which we will call "relaxed constraint system" below,

$$
\begin{array}{rlrl}
\dot{q} & =p & q(0) & =q_{0} \in \mathbb{R}^{N} \\
\dot{p} & =-G(q)^{\mathrm{T}} K \lambda-\nabla V(q) & & p(0)=p_{0}, \quad G\left(q_{0}\right) p_{0}=0 \\
g(q) & =g\left(q_{0}\right) . & &
\end{array}
$$

Remark 2.13. After differentiating the constraint, yielding $G(q) p=0, \lambda$ is given as before by (9). For $g\left(q_{0}\right)=0$ this system is equivalent to the constrained system (8) in the sense that $\mathcal{M}$ is invariant under the evolution in time following (63). Due to continuous dependency on initial data we furthermore conclude that solutions to (63) approach solutions of (8) as $g\left(q_{0}\right) \rightarrow 0$.

As pointed out previously, our method is supposed to be consistent with the unconstrained system (2) and system (8) for $\alpha=1$ and $\alpha=0$, respectively. For the first case we furthermore require high fidelity in our approximation of energy conservation for discrete solutions as well and choose the method to be symplectic [25], i.e., such that the gradient of the discrete flow $D \psi_{h}^{1}$ satisfies

$$
\left(D \psi_{h}^{1}\right)^{\mathrm{T}} J\left(D \psi_{h}^{1}\right)=J:=\left(\begin{array}{cc}
0 & \mathbb{1} \\
-\mathbb{1} & 0
\end{array}\right) .
$$

This property is shared with the analytical flow and responsible for exact conservation of the energy functional $H$ for analytical solutions as well as preservation of a modified, close by, energy functional for discrete solutions. For an extensive presentation and discussion on this topic see e.g. 25] or [35].

We build our method on the symplectic (c.f. (64) Störmer Verlet method,

$$
\begin{aligned}
q^{n+\frac{1}{2}} & =q^{n}+h p^{n} \\
p^{n+1} & \left.=p^{n}-h \nabla V\left(q^{n+\frac{1}{2}}\right)\right)-\frac{h}{\varepsilon^{2}} G^{\mathrm{T}}\left(q^{n+\frac{1}{2}}\right) K g\left(q^{n+\frac{1}{2}}\right) \\
q^{n+1} & =q^{n+\frac{1}{2}}+h p^{n+1} .
\end{aligned}
$$


Despite its simplicity this method performs exceptionally well and is extensively discussed in detail, e.g., in [25].

In contrast to (2), the constrained model equations (18) are a system of differential algebraic equations; their differentiation index is 3 [1]. Solving these equations numerically leaves the choice of either fulfilling the constraint exactly or of accepting a numerical approximation error for $g\left(q^{n}\right)=0$. In the Hamiltonian context, the first choice suggests, e.g., the SHAKE and RATTLE schemes (c.f. [35]). Given initial states $q^{n}$ and tangential momenta $p^{n}$, both algorithms use a projection to ensure $q^{n+1} \in \mathcal{M}$ and $p^{n+1} \in T_{q^{n+1}} \mathcal{M}$.

The alternative of accepting approximation errors for the constraint itself relies on index reduction of the analytical system and subsequent discretization (c.f. e.g. 1]). In this context, a common task is to design stabilized methods [2] which allow for a discrete evolution close to the constraint manifold such that the error on the constraint stays small for long times.

Since we do not aim to run the constrained model $\alpha=0$ for more than a few time steps in the blended method, we will employ an index reduction approach but ignore the issue of stabilization at this point. Motivated by the Störmer-Verlet method we propose a projection method for (8) which satisfies the hidden constraint

$$
G(q) p=0
$$

up to a given tolerance and the constraint $g(q)=0$ in (8) up to a global error of order $\mathcal{O}\left(h^{2}\right)$. The proposed discretization of the blended model (41) reads

$$
\begin{aligned}
q^{n+\frac{1}{2}} & =q^{n}+\frac{h}{2} p_{n} \\
p^{n+1, \alpha} & =p^{n}-h \nabla V\left(q^{n+\frac{1}{2}}\right)-h G^{\mathrm{T}}\left(q^{n+\frac{1}{2}}\right) K\left(\frac{\alpha}{\varepsilon^{2}} g\left(q^{n+\frac{1}{2}}\right)+(1-\alpha) \lambda^{n+\frac{1}{2}}\right) \\
q^{n+1, \alpha} & =q^{n+\frac{1}{2}}+\frac{h}{2} p^{n+1, \alpha},
\end{aligned}
$$

where $\lambda^{n+\frac{1}{2}}$ satisfies the weakly nonlinear equations

$$
\begin{aligned}
p^{n+1,0} & =p^{n}-h \nabla V\left(q^{n+\frac{1}{2}}\right)-h G^{\mathrm{T}}\left(q^{n+\frac{1}{2}}\right) K \lambda^{n+\frac{1}{2}} \\
h G\left(q^{n+\frac{1}{2}}+\frac{h}{2} p^{n+1,0}\right) G^{\mathrm{T}}\left(q^{n+\frac{1}{2}}\right) K \lambda^{n+\frac{1}{2}} & =G\left(q^{n+\frac{1}{2}}+\frac{h}{2} p^{n+1,0}\right)\left(p^{n}-h \nabla V\left(q^{n+\frac{1}{2}}\right)\right) .
\end{aligned}
$$

as derived from the relaxed constraint of tangential motion, i.e., $G(q) p=0$.

For this method, we now state and prove several consistency results:

Lemma 2.14. Let $\alpha=0$ and $\kappa \in \mathbb{N}$. Let $\left(q^{\kappa}, p^{\kappa}\right)$ be the numerical solution given by applying method (67) $\kappa$ times to initial data $\left(q^{0}, p^{0}\right) \in \mathbb{R}^{2 N}$, which satisfy $G\left(q^{0}\right) p^{0}=0$. Then $\left(q^{\kappa}, p^{\kappa}\right)$ is consistent with the analytical solution 
$(q, p)$ of the constrained system (63) at time $T=h \kappa$ for initial condition $q^{0}, p^{0}$. More specifically,

$$
\begin{aligned}
\left\|p^{\kappa}-p(T)\right\| & \leq c h \\
\left\|q^{\kappa}-q(T)\right\| & \leq \tilde{c} h^{2} \\
\left\|G\left(q^{\kappa}\right) p^{\kappa}\right\| & =0 \\
\left\|g(q(T))-g\left(q^{\kappa}\right)\right\| & \leq \hat{c} h
\end{aligned}
$$

where the constants $\tilde{c}, \hat{c}, c$ are independent of $h$ and $\kappa$.

Proof. The proof is following [36]. While first order consistency is essentially proven by a classical Taylor expansion argument, one still needs to address the algebraic constraint. At the continuous level this is readily achieved by reference to (9) which becomes

$$
K \lambda=\left(G(q) G(q)^{\mathrm{T}}\right)^{-1}\left(-G(q) \nabla V(q)+\sum_{i=1}^{L} \sum_{j=1}^{L}\left(p^{\mathrm{T}} e_{i, j} p\right) \frac{\partial^{2} g(q)}{\partial q_{i} \partial q_{j}}\right)
$$

The momentum equation in (8) is then equivalent to

$$
\dot{p}=-\mathcal{P}_{q}^{\perp} \nabla V(q)+G(q)^{\mathrm{T}}\left(G(q) G(q)^{\mathrm{T}}\right)^{-1} \sum_{i=1}^{L} \sum_{j=1}^{L}\left(p^{\mathrm{T}} e_{i, j} p\right) \frac{\partial^{2} g(q)}{\partial q_{i} \partial q_{j}} .
$$

For the discrete case we observe

$$
\begin{aligned}
G\left(q^{n+1,0}\right) p^{n} & =G\left(q^{n}\right) p^{n}+\sum_{i=0}^{N} \sum_{j=0}^{N}\left(q^{n+1,0}-q^{n}\right) e_{i, j} p^{n} \frac{\partial^{2} g\left(q^{n}\right)}{\partial q_{i} \partial q_{j}}+\mathcal{O}\left(h^{2}\right) \\
& =h \sum_{i=0}^{N} \sum_{j=0}^{N} \frac{p^{n+1,0}+p^{n}}{2} e_{i, j} p^{n} \frac{\partial^{2} g\left(q^{n+\frac{1}{2}}\right)}{\partial q_{i} \partial q_{j}}+\mathcal{O}\left(h^{2}\right),
\end{aligned}
$$

by Taylor expansion, where $G\left(q^{n}\right) p^{n}=0$ holds due to the tangential update of the previous time step or if applicable, by the initial condition in (67). Using this identity and the second and fourth update rules in (67) we can express 
$h K \lambda$ explicitly by

$$
\begin{aligned}
h K \lambda= & \left(G\left(q^{n+1,0}\right) G^{\mathrm{T}}\left(q^{n+\frac{1}{2}}\right)\right)^{-1} G\left(q^{n+1,0}\right)\left(p_{n}-h \nabla V\left(q^{n+\frac{1}{2}}\right)\right) \\
= & h\left(G\left(q^{n+1,0}\right) G^{\mathrm{T}}\left(q^{n+\frac{1}{2}}\right)\right)^{-1}\left(-G\left(q^{n+1,0}\right) \nabla V\left(q^{n+\frac{1}{2}}\right)+\left(\sum_{i=0}^{N} \sum_{j=0}^{N} \frac{p^{n+1,0}+p^{n}}{2} e_{i, j} p^{n} \frac{\partial^{2} g\left(q^{n+\frac{1}{2}}\right)}{\partial q_{i} \partial q_{j}}\right)\right) \\
& +\mathcal{O}\left(h^{2}\right) .
\end{aligned}
$$

Expanding by Taylor again, this identity now enables us to rewrite the momentum update to

$$
\begin{aligned}
p^{n+1,0}=p^{n} & -h \mathcal{P}_{q^{n+\frac{1}{2}}}^{\perp} \nabla V\left(q^{n+\frac{1}{2}}\right) \\
& -h G^{\mathrm{T}}\left(q^{n+\frac{1}{2}}\right)\left(G\left(q^{n+\frac{1}{2}}\right) G^{\mathrm{T}}\left(q^{n+\frac{1}{2}}\right)\right)^{-1} \sum_{i=0}^{N} \sum_{j=0}^{N} \frac{p^{n+1,0}+p^{n}}{2} e_{i, j} p^{n} \frac{\partial^{2} g\left(q^{n+\frac{1}{2}}\right)}{\partial q_{i} \partial q_{j}}+\mathcal{O}\left(h^{2}\right) .
\end{aligned}
$$

Comparing the momentum in closed form as given in (71) with the discretization (74), we verify second order local consistency and therefore first order global consistency

$$
\left\|p^{\kappa}-p(T)\right\| \leq c h
$$

To bound the consistency error in the coordinates one combines the update rules in (67) to

$$
q^{n+1,0}=q^{n}+h \frac{p^{n}+p^{n+1,0}}{2} .
$$

Since $p_{n+1}$ this a locally second order consistent approximation we obtain

$$
q^{n+1,0}=q^{n}+h \frac{p\left(t^{n}\right)+p\left(t^{n+1}\right)}{2}+\mathcal{O}\left(h^{3}\right)
$$

The implicit midpoint rule is of local third consistency order and we obtain

$$
\left\|q^{n+1,0}-q\left(t^{n+1}\right)\right\|=\mathcal{O}\left(h^{3}\right)
$$

and therefore

$$
\left\|q^{\kappa}-q(T)\right\| \leq \tilde{c} h^{2} .
$$


The bound for the constraint (69d) follows then directly by expanding $g(q(T))$ around $g\left(q^{n}\right)$.

Corollary 2.15. Let additionally $g\left(q^{0}\right)=0$, then $\left(q^{\kappa}, p^{\kappa}\right)$ is consistent with the constraint system (8).

So far we consider only initial data with momenta satisfying tangency to the constraint manifold, i.e., $G(q) p=0$. This is of course necessary in the context of consistency, since the underlying model is not well posed otherwise. Nevertheless the proposed usage in data assimilation procedures introduces exactly such initial data. The subsequent two statements will clarify what to expect if we apply method (67) to general initial data while $\alpha=0$.

Lemma 2.16. For $\alpha=0$ the method in (67) approximates the projection of momentum $\mathcal{P}_{q}^{\perp} p$ in the following sense.

$$
\left\|\mathcal{P}_{q^{n}}^{\perp} p^{n}-p^{n+1,0}\right\| \leq c h
$$

Proof. Use of the expression for $K \lambda$ as stated in (68b) and subsequent Taylor expansion yield

$$
\begin{aligned}
p^{n+1,0} & =p^{n}-G^{\mathrm{T}}\left(q^{n+\frac{1}{2}}\right)\left(G\left(q^{n+1,0}\right) G^{\mathrm{T}}\left(q^{n+\frac{1}{2}}\right)\right)^{-1} G\left(q^{n+1}\right) p^{n}+\mathcal{O}(h) \\
& =p^{n}-G^{\mathrm{T}}\left(q^{n}\right)\left(G\left(q^{n}\right) G^{\mathrm{T}}\left(q^{n}\right)\right)^{-1} G\left(q^{n}\right) p^{n}+\mathcal{O}(h) \\
& =\mathcal{P}_{q^{n}}^{\perp} p^{n}+\mathcal{O}(h) .
\end{aligned}
$$

Corollary 2.17. Let $\alpha=0$ and $q^{0}, p^{0} \in \mathbb{R}^{N}$, then method (67) is globally first order consistent to the solution given by the constraint system (63) and balanced initial data $q^{0}$ and $\mathcal{P}_{q^{0}}^{\perp} p^{0}$.

Next we establish a consistency result for the blending method for $\alpha \neq 0$, which will provide a connection between the discrete evolution of the blended model from (41) on the one hand, and the continuous evolution of the damped surrogate model (44) on the other hand.

Lemma 2.18. Let $\kappa \in \mathbb{N}$ and let $\alpha=\max (0,1-d h)$. Furthermore let $\left(q^{\kappa}, p^{\kappa}\right)$ be the numerical solution given by applying (67) $\kappa$ times to initial data $\left(q^{0}, p^{0}\right) \in \mathbb{R}^{2 N}$. Then $\left(q^{\kappa}, p^{\kappa}\right)$ is consistent with the solution $(q, p)$ of the dissipative system (44) at time $T=h \kappa$. More specifically,

$$
\begin{gathered}
\left\|q^{\kappa}-q(T)\right\| \leq c h^{2} \\
\left\|p^{\kappa}-p(T)\right\| \leq \tilde{c} h
\end{gathered}
$$

where $c, \tilde{c}$ is a constant independent of $h$ and $\kappa$. 
Proof. We start by expressing $\lambda$ explicitly and rewriting the momentum update as

$$
\begin{aligned}
p^{n+1, \alpha}= & p_{n}-h \nabla V\left(q^{n+\frac{1}{2}}\right)-\alpha \frac{h}{\varepsilon^{2}} G^{\mathrm{T}}\left(q^{n+\frac{1}{2}}\right) K g\left(q_{n+\frac{1}{2}}\right)-(1-\alpha) h G^{\mathrm{T}}\left(q^{n+\frac{1}{2}}\right) K \lambda \\
= & p^{n}-h \nabla V\left(q^{n+\frac{1}{2}}\right)-\alpha \frac{h}{\varepsilon^{2}} G^{\mathrm{T}}\left(q^{n+\frac{1}{2}}\right) K g\left(q^{n+\frac{1}{2}}\right) \\
& \quad-(1-\alpha) G^{\mathrm{T}}\left(q^{n+\frac{1}{2}}\right)\left(G\left(q^{n+1}\right) G^{\mathrm{T}}\left(q^{n+\frac{1}{2}}\right)\right)^{-1} G\left(q^{n+1, \alpha}\right)\left(p_{n}-h \nabla V\left(q^{n+\frac{1}{2}}\right)\right) \\
= & p^{n}-h\left(\tilde{\mathcal{P}}_{q^{n+\frac{1}{2}}}^{\perp}+\alpha \tilde{\mathcal{P}}_{q^{n+\frac{1}{2}}}\right) \nabla V\left(q^{n+\frac{1}{2}}\right)-\frac{h}{\varepsilon^{2}} \alpha G^{\mathrm{T}}\left(q^{n+\frac{1}{2}}\right) K g\left(q^{n+\frac{1}{2}}\right)-(1-\alpha) \tilde{\mathcal{P}}_{q^{n+\frac{1}{2}}} p^{n}
\end{aligned}
$$

where $\tilde{\mathcal{P}}_{q^{n+\frac{1}{2}}}=G^{\mathrm{T}}\left(q^{n+\frac{1}{2}}\right)\left(G\left(q^{n+1, \alpha}\right) G^{\mathrm{T}}\left(q^{n+\frac{1}{2}}\right)\right)^{-1} G\left(q^{n+1, \alpha}\right)$ satisfies by Taylor expansion $\tilde{\mathcal{P}}_{q_{n+\frac{1}{2}}}=\mathcal{P}_{q_{n+\frac{1}{2}}}+\mathcal{O}(h)$. For sufficiently small $h$ we now can substitute $\alpha=\max (0,1-h d)$ by $1-h d$ since $d$ is independent of $h$. Expanding by Taylor we find first order consistency with the dissipative model (44) in the sense of modified equation analysis,

$$
\begin{aligned}
p^{n+1, \alpha} & =p^{n}-h\left(\tilde{\mathcal{P}}_{q^{n+\frac{1}{2}}}^{\perp}+(1-h d) \tilde{\mathcal{P}}_{q^{n+\frac{1}{2}}}\right) \nabla V\left(q^{n+\frac{1}{2}}\right)-\frac{h}{\varepsilon^{2}}(1-h d) G^{\mathrm{T}}\left(q^{n+\frac{1}{2}}\right) K g\left(q^{n+\frac{1}{2}}\right)-h d \tilde{\mathcal{P}}_{q^{n+\frac{1}{2}}} p_{n} \\
& =p^{n}-h\left(\varepsilon^{-2} G^{\mathrm{T}}\left(q^{n+\frac{1}{2}}\right) K g\left(q^{n+\frac{1}{2}}\right)+d \mathcal{P}_{q^{n+\frac{1}{2}}} p^{n}+\nabla V\left(q^{n+\frac{1}{2}}\right)\right)+\mathcal{O}\left(h^{2}\right) .
\end{aligned}
$$

The update of the coordinates can be rewritten as

$$
q^{n+1}=q^{n}+h \frac{p^{n}+p^{n+1}}{2}
$$

and therefore the same proof as in Lemma 2.14 leads to the statement.

Remark 2.19. Although it may seem odd at a first glance that we have to change $\alpha$ depending on $h$ to achieve convergence to solutions of the dissipative surrogate model, this does not contradict the fact that given an a priori choice of $\alpha$, discrete solutions of the blended method are consistent approximations to solutions of system (44) for a certain $d$. Thus, the discretized blended model inherits the tendency to approach the balanced manifold from the dissipative model as desired. The relationship between $\alpha, d$, and $h$ is also what motivates our reference to the modified equation approach.

To complete and illustrate the overall picture we collect most of the preceding results and references for the proof of the following commuting diagram.

Proposition 2.20. Let $\alpha=\max (0,1-h d)$. Let $\phi$ and $\psi$ be the analytical and numerical flows respectively, with regard to the models as mentioned below, then the diagram in Figure [6] commutes.

Proof. The consistency of the Störmer-Verlet method is stated in e.g. 25] and for an overview of all the other 


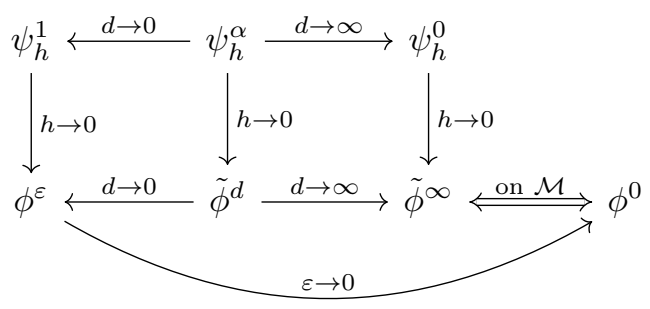

Figure 6: The commuting diagram shows the connections between the analytical model hierarchy given by the flow $\phi^{\epsilon}$ of the Hamiltonian system (2), the flow $\tilde{\phi}^{d}$ of the dissipative system (44) and the flow $\tilde{\phi}^{\infty}$ of the relaxed constrained model (63). Furthermore the diagram depicts the consistency results, of the Störmer-Verlet method (65) and the blended method (42) denoted by $\psi_{h}^{\alpha}$. The flow for the constrained model (8) is denoted by $\psi^{0}$.

connections in the commuting diagram in Figure 6 we refer to Figure 7 .

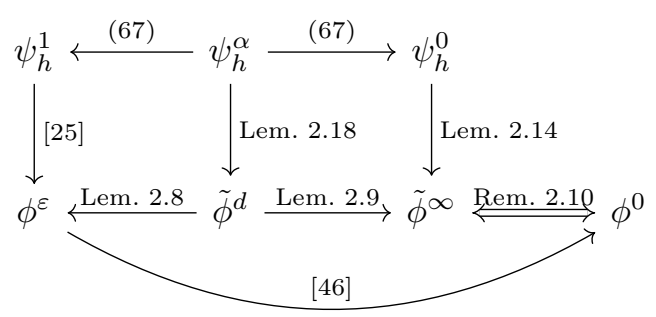

Figure 7: The diagram depicts the same situation as in Figure 6, but refers to the previously established results and relevant literature, instead of the limits.

\section{$3 \quad$ Numerical Results}

For experiments in the context of data assimilation one immediate obstacle arises from potential model errors. We avoid this question by considering an initially balanced reference solution of (2) which is approximated by the Störmer-Verlet method (65). Henceforth this solution will be denoted by $z^{\text {ref }}$. The observations then are given by $y_{\mathrm{obs}}\left(t_{k}\right)=H_{\mathrm{obs}} z^{\mathrm{ref}}\left(t_{k}\right)+\zeta_{k}$. Hereby $H_{\mathrm{obs}} z\left(t_{k}\right)=q\left(t_{k}\right)$ and $\zeta_{k}$ is the realization of the normally distributed measurement error at some time $t_{k}=k \Delta t_{\mathrm{obs}}$, when the observation becomes available. We assume the measurement error to have zero mean and covariance $R=\rho I$. The resulting evolution of observations is assimilated by the proposed data assimilation scheme. The advantage of this setup is the straightforward assessment of the quality of the data assimilation method by comparing the reference solution to, e.g., the ensemble members or the point estimate of their mean.

According to (c.f. [45]), due to finite ensemble sizes the true covariances of the posterior distributions are underestimated in ensemble based data assimilation methods. One technique to address this issue is ensemble 
Balanced data assimilation

inflation which amounts to an artificial increase of the spread of the ensemble after each assimilation step by

$$
z_{i}^{\text {new }}:=\bar{z}+\sigma_{\text {infl }}\left(z_{i}-\bar{z}\right)
$$

In the experiments we apply the ensemble inflation as the last step of the assimilation procedure. For the comparison of the presented methods we choose again the stiff elastic double pendulum from Example 1.4 as the dynamical model. The initial ensemble is constructed from copies of the initial state of $z^{\text {ref }}$ except for the components tangential to the constraint manifold $\mathcal{M}$. For those we perturb the reference state normally distributed with covariance $\rho_{0}$. Subsequently we eliminate the resulting normal component i.e. balance the initial data by minimizing (25a) for $B=0$ and $\gamma=0$. This is a natural modification of the penalty method for the first step and ensures the samples are spread along the constraint manifold only. In comparing the different balancing methods, the initial data are always generated in the way just described.

For the blended time stepping method we choose a linear ramp for $\alpha$ as depicted in Fig. 5 where $\alpha=0$ initially and $\alpha=1$ at the end of the blending window. The analysis of the damped model equation in (44) is based on linearization and suggests that, similarly to the situation with the harmonic oscillator, one can find values of the blending and damping parameters $\alpha$ and $d$, respectively, that imply dynamics close to the aperiodic case. As we do not further investigate the question for optimal $\alpha$ we choose a linear ramp to step through different values of the damping coefficient as brute force approach.

For the numerical values of the parameters of the experiments we refer to Table 1. As with regard to the

\begin{tabular}{cccccccccccccc}
\hline$B$ & $L$ & $l$ & $\varepsilon$ & $K$ & $a_{0}$ & $\Delta t$ & $M$ & $H_{\text {obs }} z$ & $\Delta t_{\text {obs }}$ & $\rho$ & $\rho_{0}$ & $\sigma_{\text {inf }}$ & $T$ \\
\hline $\mathbb{1}$ & 2 & $(1,1)$ & 0.001 & $\operatorname{diag}(1,0.04)$ & 9.81 & 0.001 & 20 & $q$ & 0.1 & 0.05 & 0.05 & 1.05 & 500 \\
\hline
\end{tabular}

Table 1: Parameters for the numerical experiments using the double pendulum model.The model parameters are given by the equilibrium lengths $l \in \mathbb{R}^{L}$, the scale separation parameter $\varepsilon$, the stiffness matrix $K$ and the gravity $a_{0}$. The model is discretized by the Störmer-Verlet method (65) with step width $\Delta t . \quad M$ denotes the ensemble size, $\Delta t_{\text {obs }}$ the interval between two observations and $H z$ the observed variable. We choose $\rho$ as covariance of the measurement error, $\rho_{0}$ as the initial uncertainty i.e. the covariance of the initial ensemble and $\rho_{\text {inf }}$ as the inflation factor. Finally $T$ denotes the duration of the experiment.

implementation details, we minimize the functional (25a) using either the Broyden-Fletcher-Goldfarb-Shanno (BFGS) [10, 19, 21, 47] method as implemented in scipy 49] or the proposed algorithm of (33). For the first we require a tolerance of $10^{-8}$ and as initial values we choose the results of the plain EnKF. In the second case we choose a fixed step size of $h=10^{-3}$ and iterate as long as the maximal absolute value of the increment (31) exceeds $10^{-8}$. Additionally we need to solve a nonlinear system for the implicit part of the blended time stepping method (67) when $\alpha=0$. This system is solved using the scipy [49] wrapper for the modified Powell method from 
the MINPACK [42] subroutine hybrd. The initial value is the zero vector of dimension $L$ and the tolerance for the nonlinear problem is set to double precision i.e. $10^{-16}$. To quantify the error of the methods we use the time averaged root mean square error as given in [45]

$$
\operatorname{TRMSE}(Z)=\sqrt{\frac{1}{N_{T}} \sum_{i}^{N_{T}}\left\|\hat{Z}\left(t_{i}\right)-Z\left(t_{i}\right)\right\|^{2}} .
$$

Hereby $\hat{Z}$ denotes the estimate for the quantity $Z$ and both are evaluated at $N_{T}$ time points $t_{k} \in[0, T]$.

This score is shown below as a function of the tuning parameters of the respective method. For comparison we furthermore show the results for the unmodified ensemble Kalman filter. As seen in Figures $8-10$, the forecast quality for the coordinates and the momenta improve drastically when choosing appropriate tuning parameters for the respective methods.

For the penalty method we realize from Figures 8 and 9 that we obtain the best results when forcing the analysis balance residual of each ensemble member to be close to the respective one inferred from the forecast. We can enforce this by the penalty method when setting $\gamma=1$. We also find that increased weights do add to the forecast quality only up to certain extent.

The blending method only allows for one tuning parameter, the blending window size. Comparing several choices in Figure 10, we obtain the best results when choosing a window large enough to capture a full period of the less stiff spring in the blending window. This happens approximately around $\frac{2 \pi \varepsilon}{\sqrt{k_{2}}} \approx 0.3 \Delta t_{\mathrm{obs}}$.

\section{Conclusions}

Aiming to improve data assimilation for slow solutions of highly oscillatory systems, this paper suggests two principally different extensions of ensemble-based data assimilation algorithms. The first approach modifies the data assimilation scheme itself and consists of a rather generic post processing step involving the minimization of a cost functional that quantifies the oscillatory solution content.

The second approach utilizes the ability of asymptotically consistent numerical schemes which provide seamless access to both the highly oscillatory systems of interest and to the reduced differential-algebraic counterparts describing motions on the associated slow manifolds. Following ideas first formulated in [3], this method filters oscillatory, off-manifold, components arising in the course of an assimilation step by starting the subsequent forward simulation with a few time steps of the reduced dynamics and blending the solver back to the full system over another couple of time steps. Whereas the full and reduced dynamics are represented by (nearly) energy preserving 

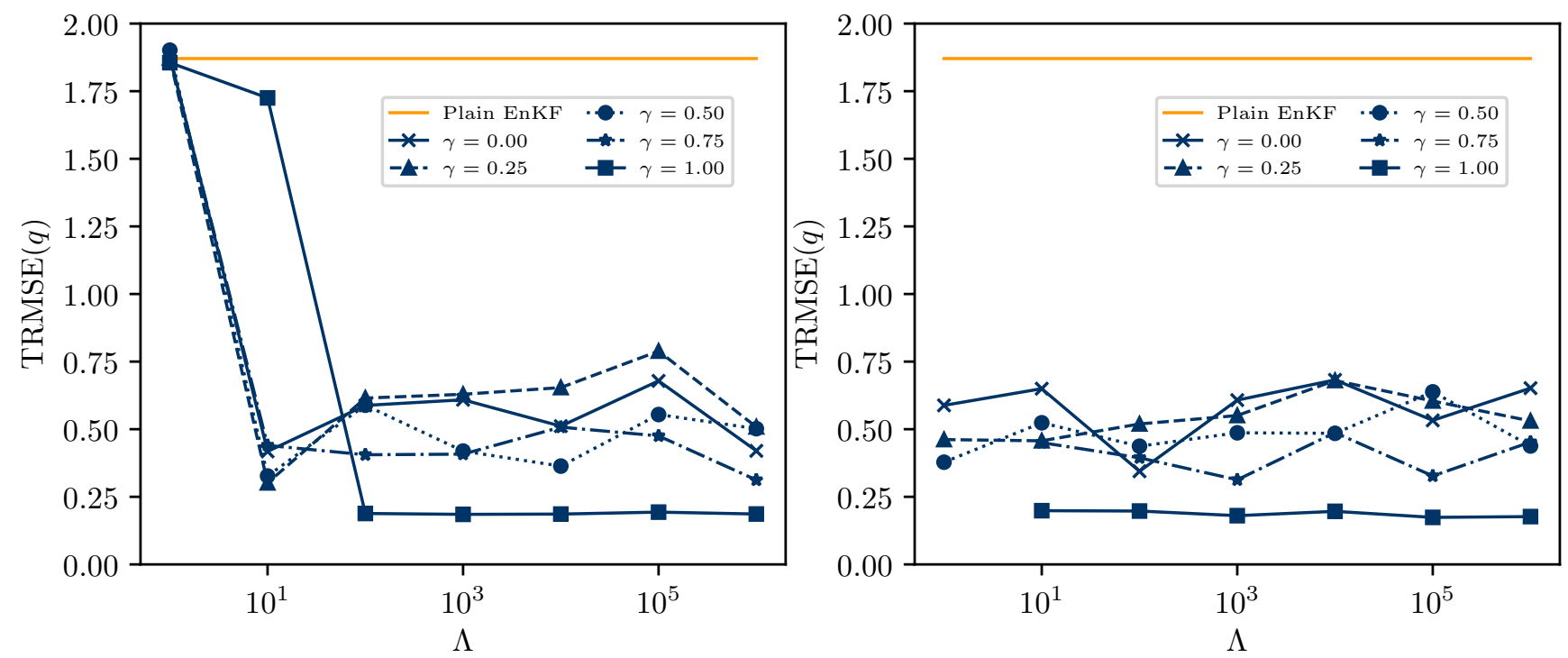

Figure 8: The left panel depicts the time averaged root mean square error (TMRSE) of the coordinates obtained by the penalty method minimizing the functional (25a) and using the previously mentioned BFGS solver. The right panel shows the results for the same experiment, but using the penalty method solved by the descent with modified search direction from (33). In orange we depict the results obtained by the unmodified ensemble Kalman filter.
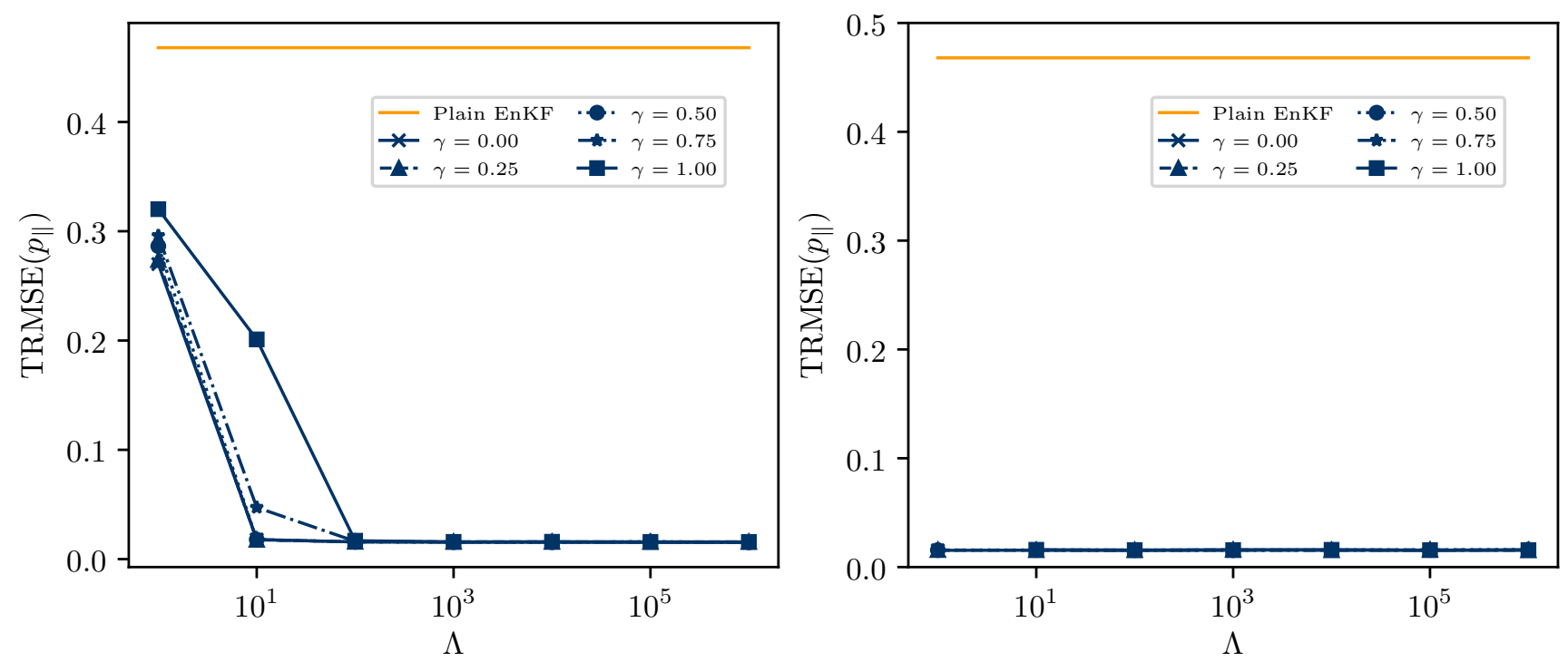

Figure 9: The left and right panels show the time averaged root mean square error in the tangential component of the unobserved momenta, for the penalty method solved by the BFGS and again (33) respectively. In orange we depict the results obtained by the unmodified ensemble Kalman filter. 

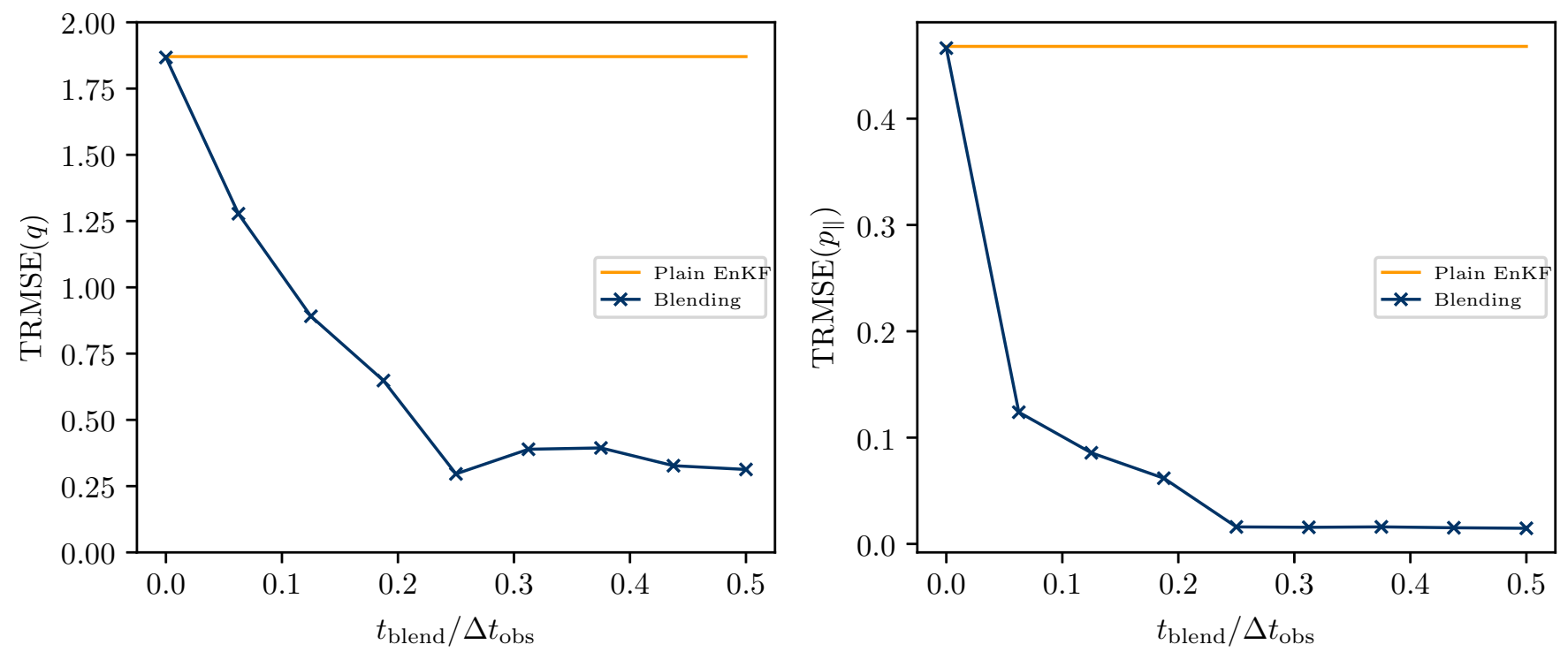

Figure 10: The left panel shows the time averaged root mean square error for the coordinates obtained by the blending method. The right panel displays the same for the tangential component of the unobserved momenta. For comparison the results obtained by the unmodified ensemble Kalman filter are shown in orange. The abscissa describes the ratio of the length of the blending window and the observation interval.

integrators, the intermediate systems accessed during the blending phase are designed here to selectively dissipate the oscillatory solution components. This prohibits the re-introduction of oscillations in the course of the blending procedure. A rigorous justification of the blended time-stepping method by asymptotic analysis is provided. The optimal parameter choice within the blending time window remains a topic for further investigation.

It is demonstrated that both methods perform well in terms of forecast quality and allow accurate state estimation in situations where the standard ensemble Kalman Filter fails to do so. The dependency of the forecast skill on the respective tuning parameters behaves as expected in our prototypical test case of the elastic double pendulum. Both approaches leave room for further improvement and extension, however. Thus, e.g., seamless incorporation of the balancing step in a Bayesian filter may help optimizing both in terms of accuracy, balancing quality, and efficiency. Also, as one referee of this paper pointed out, the implicit particle filter technique of A. Chorin and coworkers, [13, 14], may provide a means of effectively steering an entire ensemble to predominantly sample balanced states. This would be achieved by penalizing the probability of unbalanced states within the space of probability distributions which the method has access to. For the blending approach, aside from optimized sequences of the blending parameter, additional gains are conceivable when the fast part of the dynamics is known to be linear, as is the case in atmospheric flow applications.

The broader application area for the two proposed stabilization techniques is ensemble-based data assimilation 
for geophysical processes. This application area shares the situation of small oscillatory energy and conservative motion along a slow manifold. Ongoing research therefore investigates the efficacy of the proposed methods for data assimilation into multidimensional geophysical flow models. Depending on the pertinent spatio-temporal scales, several different dominant balances emerge in those models [32], with geostrophic balance as a prominent example. In contrast to the present work, these balances are often essentially linear so that Lemma 1.7 applies and only weak generation of imbalances by the standard EnKF is expected. Considerable imbalances are introduced, however, by spatial localization in the assimilation algorithm, a measure that is used to avoid artificial global scale correlations. Localization destroys the linearity of the filter transformation and therefore gives rise to stronger imbalances again. The methodologies proposed here both directly translate to this context, since neither of the algorithms leverages the linearity of the filter. Ongoing studies investigate these issues for the rotational shallow water equations as well as for a vertical slice model of the atmosphere.

\section{Acknowledgments}

This research has been partially funded by Deutsche Forschungsgemeinschaft (DFG) through grant CRC 1114 "Scaling Cascades in Complex Systems", Project Number 235221301, Project A02 "Multiscale data and asymptotic model assimilation for atmospheric flows".

\section{References}

[1] U. M. Ascher and L. R. Petzold. Computer Methods for Ordinary Differential Equations and DifferentialAlgebraic Equations. Society for Industrial and Applied Mathematics, Philadelphia, 1998.

[2] U. M. Ascher, H. Chin, and S. Reich. Stabilization of DAEs and invariant manifolds. Numerische Mathematik, $67(2): 131-149,1994$.

[3] T. Benacchio, W. P. O’Neill, and R. Klein. A blended soundproof-to-compressible numerical model for smallto mesoscale atmospheric dynamics. Monthly Weather Review, 142(12):4416-4438, 2014.

[4] G. Benettin, L. Galgani, and A. Giorgilli. Realization of holonomic constraints and freezing of high frequency degrees of freedom in the light of classical perturbation theory .1. Communications in Mathematical Physics, 113(1):87-103, 1987.

[5] G. Benettin, L. Galgani, and A. Giorgilli. Realization of holonomic constraints and freezing of high frequency 
degrees of freedom in the light of classical perturbation theory .2. Communications in Mathematical Physics, 121(4):557-601, 1989.

[6] K. Bergemann and S. Reich. A mollified ensemble Kalman filter. Quarterly Journal of the Royal Meteorological Society, 136:1636-1643, 2010.

[7] S. Bloom, L. L. Takacs, A. Da Silva, and D. Ledvina. Data assimilation using incremental analysis updates. Monthly Weather Review, 124:1256-1271, 1996.

[8] O. Bokhove and T. G. Shepherd. On hamiltonian balanced dynamics and the slowest invariant manifold. Journal of the Atmospheric Sciences, 53(2):276-297, 1996.

[9] F. A. Bornemann and C. Schütte. Homogenization of Hamiltonian systems with a strong constraining potential. Physica D, 102(1-2):57-77, 1997.

[10] C. G. Broyden. The Convergence of a Class of Double-rank Minimization Algorithms 1. General Considerations. IMA Journal of Applied Mathematics, 6(1):76-90, 1970.

[11] R. Camassa. On the geometry of an atmospheric slow manifold. Physica D: Nonlinear Phenomena, 84(3-4): 357-397, 1995.

[12] A. J. Chorin. The numerical solution of Navier-Stokes equations for an imcompressible fluid. Bulletin of the American Mathematical Society, 73(6):928-931, 1967.

[13] A. J. Chorin and X. Tu. Implicit sampling for particle filters. P. Natl. Acad. Sci. USA, 106(41):17249-17254, 2009.

[14] A. J. Chorin, M. Morzfeld, and X. Tu. Implicit particle filters for data assimilation. Comm. Appl. Math. Comput. Sci., 5(2):221-240, 2010.

[15] F. Cordier, P. Degond, and A. Kumbaro. An Asymptotic-Preserving all-speed scheme for the Euler and Navier-Stokes equations. J. Comput. Phys., 231:5685-5704, 2012.

[16] C. Cotter. Data assimilation on the exponentially accurate slow manifold. Philosophical Transactions of the Royal Society A: Mathematical, Physical and Engineering Sciences, 371(1991):20120300, 2013.

[17] G. Evensen. The Ensemble Kalman Filter: theoretical formulation and practical implementation. Ocean Dynamics, 53:343, 2003. 
[18] N. Fenichel. Geometric singular perturbation theory for ordinary differential equations. Journal of Differential Equations, 31(1):53-98, 1979.

[19] R. Fletcher. A new approach to variable metric algorithms. The Computer Journal, 13(3):317-322, 1970.

[20] C. W. Gear. Maintaining solution invariants in the numerical solution of odes. SIAM J. Sci. Stat. Comput., 7 (3):734-743, 1986.

[21] D. Goldfarb. A family of variable-metric methods derived by variational means. Mathematics of Computation, 24(109):23-23, 1970.

[22] G. H. Golub and C. F. Van Loan. Matrix Computations. Johns Hopkins Studies in the Mathematical Sciences. Johns Hopkins University Press, Baltimore, 3rd ed edition, 1996.

[23] G. A. Gottwald. Controlling balance in an ensemble Kalman filter. Nonlinear Processes in Geophysics, 21(2): 417-426, 2014.

[24] G. A. Gottwald, L. Mitchell, and S. Reich. Controlling overestimation of error covariance in ensemble kalman filters with sparse observations: A variance-limiting kalman filter. Monthly Weather Review, 139(8):2650-2667, 2011. doi: 10.1175/2011MWR3557.1. URL https://journals.ametsoc.org/view/journals/mwre/139/8/2011mwr3557.1.xml

[25] E. Hairer, C. Lubich, and G. Wanner. Geometric numerical integration: structure-preserving algorithms for ordinary differential equations. Springer series in computational mathematics. Springer, Berlin u.a., 2. edition, 2010.

[26] J. Harlim and A. J. Majda. Catastrophic filter divergence in filtering nonlinear dissipative systems. Communications in Mathematical Sciences, 8(1):27-43, 2010.

[27] S. Jin. Asymptotic preserving (ap) schemes for multiscale kinetic and hyperbolic equations: a review. Riv. Mat. Univ. Parma, 3:177-216, 2012.

[28] R. E. Kalman. A new approach to linear filtering and prediction problems. Journal of Basic Engineering, 82: $35,1960$.

[29] E. Kalnay. Atmospheric Modeling, Data Assimilation and Predictability. Cambridge University Press, 1 edition, 2002. 
[30] D. Kelly, A. J. Majda, and X. T. Tong. Concrete ensemble Kalman filters with rigorous catastrophic filter divergence. P. Natl. Acad. Sci. USA, 112(34):10589-10594, 2015.

[31] J. D. Kepert. Covariance localisation and balance in an Ensemble Kalman Filter. Quarterly Journal of the Royal Meteorological Society, 135(642):1157-1176, 2009.

[32] R. Klein. Scale-dependent asymptotic models for atmospheric flows. Ann. Rev. Fluid Mech., 42:249-274, 2010.

[33] R. Klein, N. Botta, L. Hofmann, A. Meister, C. Munz, S. Roller, and T. Sonar. Asymptotic adaptive methods for multiscale problems in fluid mechanics. J. Engrg. Math., 39:261-343, 2001.

[34] C. Kuehn. Geometric Singular Perturbation Theory, pages 53-70. Springer International Publishing, Cham, 2015 .

[35] B. Leimkuhler and S. Reich. Simulating Hamiltonian Dynamics. Cambridge Monographs on Applied and Computational Mathematics. Cambridge University Press, 2005.

[36] B. J. Leimkuhler and R. D. Skeel. Symplectic Numerical Integrators in Constrained Hamiltonian Systems. Journal of Computational Physics, 112(1):117-125, 1994.

[37] E. N. Lorenz. Deterministic nonperiodic flow. Journal of the Atmospheric Sciences, 20(2):130-141, 1963.

[38] E. N. Lorenz. Predictability - a problem partly solved. In T. Palmer and R. Hagedorn, editors, Predictability of Weather and Climate, pages 40-58. Cambridge University Press, 2006.

[39] P. Lynch. The swinging spring: a simple model for atmospheric balance. In J. Norbury and I. Roulstone, editors, Large-Scale Atmosphere-Ocean Dynamics: Volume II: Geometric Methods and Models, page 64, 2002.

[40] P. Lynch. The Emergence of Numerical Weather Prediction: Richardson's Dream. Cambridge University Press, 2014 .

[41] P. Lynch and X.-Y. Huang. Initialization of the HIRLAM model using a digital filter. Monthly Weather Review, 120:1019-1034, 1992.

[42] J. J. Moré, B. S. Garbow, and K. E. Hillstrom. User guide for MINPACK-1. Technical Report ANL-80-74, Argonne Nat. Lab., Argonne, IL, 1980.

[43] S. Reich. Smoothed dynamics of highly oscillatory Hamiltonian systems. Physica D, 89:28—42, 1995.

[44] S. Reich. Smoothed Langevin dynamics of highly oscillatory systems. Physica D, 138:210-224, 2000. 
[45] S. Reich and C. Cotter. Probabilistic forecasting and Bayesian data assimilation: a tutorial. Cambridge University Press, 2015.

[46] H. Rubin and P. Ungar. Motion under a Strong Constraining Force. Communications on Pure and Applied Mathematics, 10(1):65-87, 1957.

[47] D. F. Shanno. Conditioning of quasi-Newton methods for function minimization. Mathematics of Computation, 24(111):647-647, 1970.

[48] F. Takens. Motion under the influence of a strong constraining force. Springer Berlin Heidelberg, 1980.

[49] P. Virtanen, R. Gommers, T. E. Oliphant, M. Haberland, T. Reddy, D. Cournapeau, E. Burovski, P. Peterson, W. Weckesser, J. Bright, S. J. van der Walt, M. Brett, J. Wilson, K. Jarrod Millman, N. Mayorov, A. R. J. Nelson, E. Jones, R. Kern, E. Larson, C. Carey, İ. Polat, Y. Feng, E. W. Moore, J. VanderPlas, D. Laxalde, J. Perktold, R. Cimrman, I. Henriksen, E. A. Quintero, C. R. Harris, A. M. Archibald, A. H. Ribeiro, F. Pedregosa, P. van Mulbregt, and S. . Contributors. SciPy 1.0: Fundamental Algorithms for Scientific Computing in Python. Nature Methods, 17:261-272, 2020.

[50] R. F. Warming and F. Hyett. The modified equation approach to the stability and accuracy analysis of finite difference methods. J. Comput. Phys, 14(2):159-179, 1974.

[51] J. S. Whitaker and T. M. Hamill. Ensemble Data Assimilation without Perturbed Observations. Monthly Weather Review, 130(7):1913-1924, July 2002. ISSN 0027-0644.

[52] J. Zhou, S. Reich, and B. Brooks. Elastic molecular dynamics with self-consistent flexible constraints. J. Chem. Phys., 112:7919-7929, 2000. 